\title{
44. LATE QUATERNARY CARBONATE AND ISOTOPE STRATIGRAPHY, SUBANTARCTIC SITE 594, SOUTHWEST PACIFIC1
}

\author{
Campbell S. Nelson, Chris H. Hendy, Alison M. Cuthbertson, and Grant R. Jarrett, University of Waikato, \\ Hamilton, New Zealand ${ }^{2}$
}

\begin{abstract}
The late Quaternary sequence off eastern South Island, New Zealand, consists of $\sim 100 \mathrm{~m}$ of alternating bluish gray pelagic oozes and greenish gray hemipelagic oozes that extend uninterruptedly back to the Brunhes/Matuyama boundary $(0.73 \mathrm{~m} . \mathrm{y}$.$) . A very high resolution (\sim 2400 \mathrm{yr}$.) record of sediment texture, calcium carbonate content, and planktonic and benthic foraminiferal oxygen and carbon isotope composition demonstrates an in-phase cyclical fluctuation between the sedimentary parameters that closely correspond to the pelagic-hemipelagic sedimentation cycles and the isotope composition. Pelagic oozes, formed during interglacial periods of high eustatic sea level, are characterized by calcareous microfossils, relative enrichment in sand and clay sizes, high carbonate contents, reduced $\delta^{18} \mathrm{O}$ values, and increased $\delta^{13} \mathrm{C}$ values. Hemipelagic oozes, associated with glacial episodes and lowered eustatic sea level, include common terrigenous material and siliceous microfossils, are enriched in silt sizes, have low carbonate contents, high $\delta^{18} \mathrm{O}$ values, and low $\delta^{13}$ values. The history of alpine glaciations and associated erosion of the South Island of New Zealand, as expressed by the appearance of hemipelagic oozes, can be correlated directly with the major fluctuations of Northern Hemisphere ice sheets as expressed by the influence of eustatic sea-level changes on the oxygen isotope composition of both planktonic and benthic foraminifers. This high-accumulation-rate record contains conspicuous intervals of highfrequency, high-amplitude isotope variability including the presence of multiple glacial/interglacial intervals within single isotope stages, and offers one of the best sections cored to date for detailed study of the evolution and history of climate change over the last $0.75 \mathrm{~m} . \mathrm{y}$.
\end{abstract}

\section{INTRODUCTION}

Site $594\left(45^{\circ} 31.41^{\prime} \mathrm{S}, 174^{\circ} 56.88^{\prime} \mathrm{E}\right)$ is located $300 \mathrm{~km}$ east of South Island, New Zealand, on the southern margin of Chatham Rise in a water depth of $1204 \mathrm{~m}$ (Fig. 1). The site lies in the subantarctic water mass immediately south of the Subtropical Convergence and is the southernmost site of a north-south transect (Sites 586594) drilled on Leg 90 in the southwest Pacific. Because of its proximity to the New Zealand landmass, sedimentation at the site has been influenced by both oceanic and terrigenous processes. In particular, the 170-m-thick Pliocene-Quaternary interval, drilled with almost $100 \%$ recovery using the hydraulic piston corer, comprises an alternating sequence of pelagic and hemipelagic sediments (Fig. 2) whose major characteristics are listed and contrasted in Table 1 . The sedimentary cycles are probably sensitive indicators of glacial-interglacial climate fluctuations (Griggs et al., 1983) with the pelagic lithofacies forming during interglacial periods and the hemipelagic lithofacies during glacial episodes. If this is true, then Site 594 offers an extremely detailed oceanic record of climate change through the latest Cenozoic with excellent potential for correlation with the continental and marine Plio-Pleistocene and Holocene sequences of New Zealand.

The climate signature contained in the alternating pelagic-hemipelagic sedimentary sequence is currently be-

\footnotetext{
${ }^{1}$ Kennett, J. P., von der Borch, C. C., et al., Init. Repts. DSDP, 90: Washington (U.S. Govt, Printing Office)

2 Addresses: (Nelson, Jarrett) Department of Earth Sciences, University of Waikato, Hamilton, New Zealand; (Hendy, Cuthbertson) Department of Chemistry, University of Waikato, Hamilton, New Zealand.
}

ing investigated using three approaches: sediment particle-size characteristics; calcium carbonate content; and oxygen and carbon stable isotope analysis of planktonic (Globigerina bulloides d'Orbigny) and benthic (Uvigerina or Cibicides spp.) foraminifers. Details of analytical procedures are described fully by Nelson, Hendy, and Dudley (this volume), although here the content of silt$(4-63 \mu \mathrm{m})$ and clay- $(<4 \mu \mathrm{m})$ sized particles in samples has been determined automatically using a Shimadzu Centrifugal Particle Size Analyzer SA-CP2 (Healy et al., 1983), and the calcium carbonate values were determined by back titration following sample acidification using a Mettler DL40RC automatic titrator.

\section{RESULTS}

This chapter is only a preview of some of the sedimentary and isotope results obtained to date on the late Quaternary samples from Site 594. The data are given in Table 2 and are summarized on a stratigraphic log (Fig. 3) which extends back to straddle the Brunhes/Matuyama boundary $(0.73 \mathrm{~m} . \mathrm{y}$.$) at a sub-bottom depth of 99.3 \mathrm{~m}$.

Some biostratigraphic data pertinent to age control within the sequence are summarized in Table 3 . The anomalous occurrence of the calcareous nannofossil Calcidiscus macintyrei at the bottom of the sequence is explanable in terms of sediment reworking associated with formation of the major Matuyama disconformity $(\sim 1.6$ to $\sim 0.74 \mathrm{~m}$.y.) immediately below the Brunhes/Matuyama boundary (Site 594 report, this volume). The last occurrence of both the radiolarian Stylatractus universus and the calcareous nannofossil Pseudoemiliania lacunosa is at about $54 \mathrm{~m}$ sub-bottom in an interval corresponding to one of the most glacial periods in the isotope record (Fig. 3). Elsewhere, these bioevents have been 


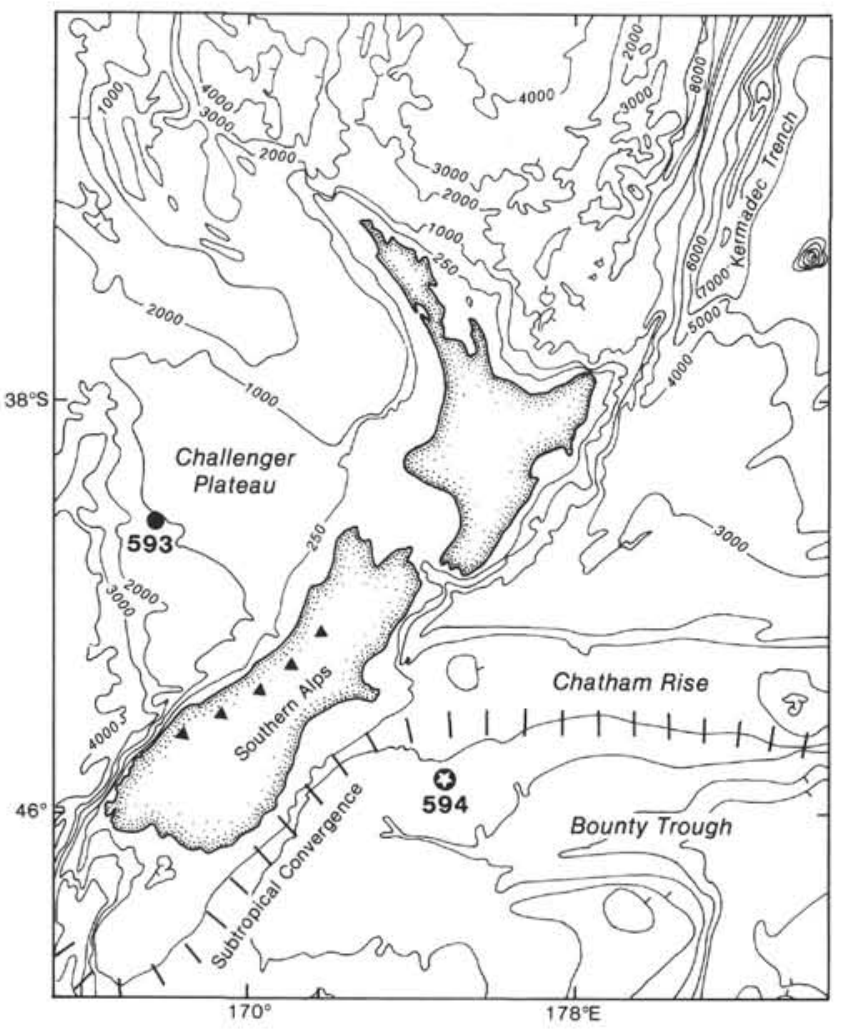

Figure 1. Location of Site 594 off southeastern New Zealand showing also some relevant physiographic features and the approximate position of the Subtropical Convergence.
Table 1. General sediment properties of the late Quaternary pelagic and hemipelagic oozes at Site 594.

\begin{tabular}{|c|c|c|}
\hline Property & Pelagic lithofacies & Hemipelagic lithofacies \\
\hline Color & $\begin{array}{l}\text { Bluish gray ( } 5 \mathrm{~B} 7 / 1 \text { to } 5 \mathrm{~B} \\
5 / 1 \text { ) }\end{array}$ & $\begin{array}{l}\text { Greenish gray ( } 5 G 6 / 1 \text { to } \\
5 G 4 / 1)\end{array}$ \\
\hline \multicolumn{3}{|l|}{ Grain size } \\
\hline Sand & Rare to common & Rare \\
\hline Silt & Abundant & Abundant \\
\hline Clay & Common to abundant & Rare to common \\
\hline $\mathrm{CaCO}_{3}$ content & Moderate to very high & Very low to moderate \\
\hline Nannofossils & Abundant & Rare to abundant \\
\hline Foraminifers & Common & Rare \\
\hline $\begin{array}{l}\text { Siliceous } \\
\text { microfossils }\end{array}$ & Absent to rare & Rare to common \\
\hline $\begin{array}{l}\text { Terrigenous } \\
\text { sediment }\end{array}$ & Rare to common & Common to abundant \\
\hline $\begin{array}{l}\text { Dominant } \\
\text { sediment } \\
\text { types }\end{array}$ & $\begin{array}{l}\text { Foraminiferal-bearing } \\
\text { nannofossil ooze; } \\
\text { foraminifer- and clay- } \\
\text { bearing nannofossil } \\
\text { ooze; foraminifer- } \\
\text { bearing clayey nanno- } \\
\text { fossil ooze }\end{array}$ & $\begin{array}{l}\text { Clay-, silt-, and/or diatom- } \\
\text { bearing nannofossil } \\
\text { ooze; nannofossil-, } \\
\text { diatom-, and/or } \\
\text { sponge-spicule-bearing } \\
\text { clayey silt }\end{array}$ \\
\hline
\end{tabular}

shown to have occurred between $\sim 0.41$ and 0.46 m.y. ago, typically within isotope stage 12 (Hays and Shackleton, 1976; Gartner, 1977; Shackleton, 1977b; Thierstein et al., 1977) which is characterized by extreme ${ }^{18} \mathrm{O}$ enrichment (e.g., Prell, 1982; Imbrie et al., in press). About $10 \mathrm{~m}$ above this level a few specimens of the calcareous nannofossil Emiliania huxleyi are recorded by Lohman (this volume), although the taxon does not become common until $37 \mathrm{~m}$ sub-bottom and abundant un-
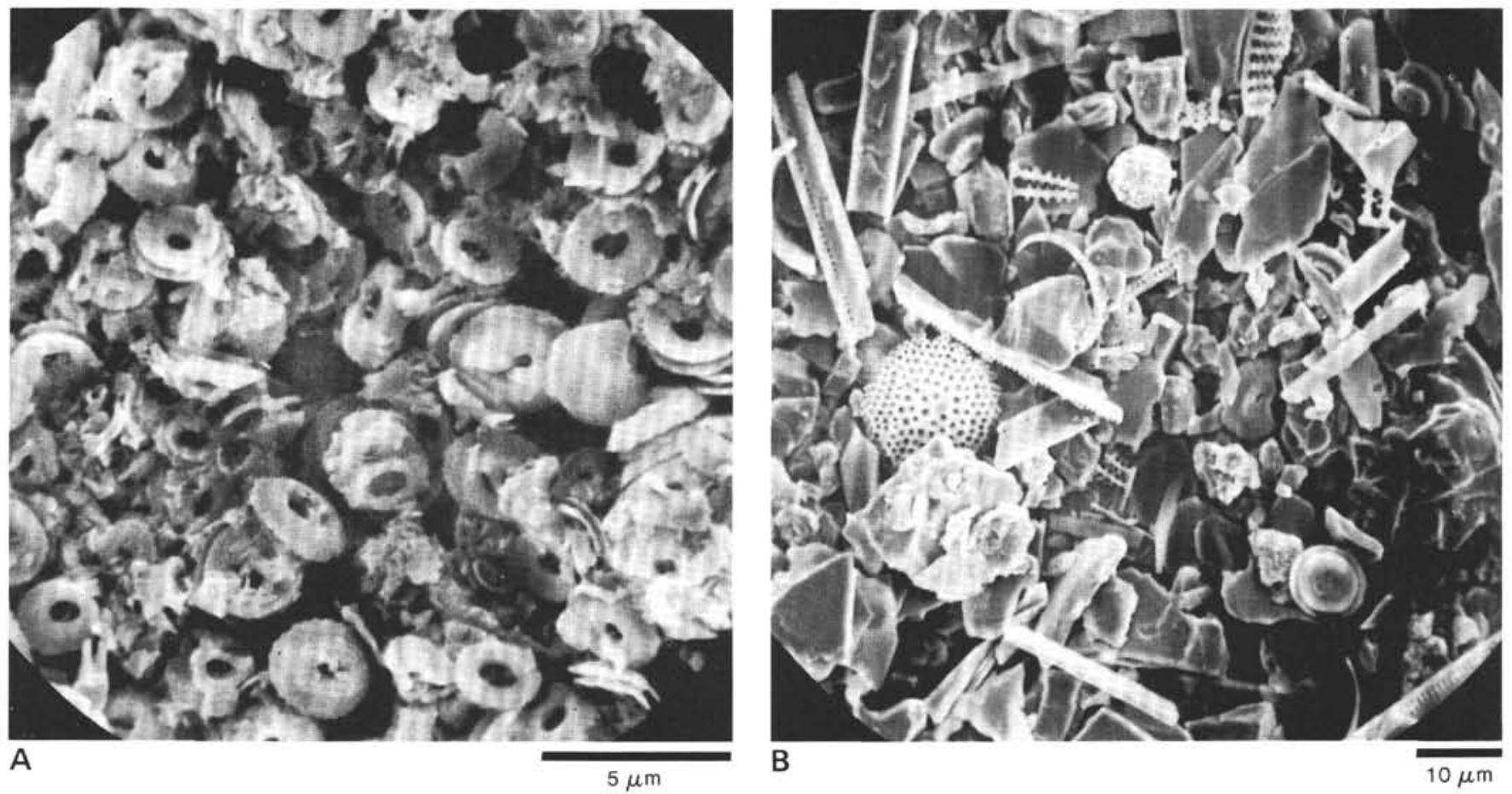

Figure 2. Scanning electron microscope images showing the compositional contrast between (A) typical pelagic and (B) hemipelagic sediments in the late Quaternary sequence at Site 594 . The pelagic sediment is dominated here by calcareous nannofossils and the hemipelagic sediment by siliceous microfossil material and terrigenous silt grains of mainly mica and quartz. 
Table 2. Carbon and oxygen data (per mil deviations from the PDB standard) for foraminifers, and calcium carbonate and textural (sand, $>63 \mu \mathrm{m}$; silt, 4-63 $\mu \mathrm{m}$; clay, $<4 \mu \mathrm{m}$ ) data for bulk samples from the late Quaternary interval of Holes 594 and 594A.

\begin{tabular}{|c|c|c|c|c|c|}
\hline \multirow{2}{*}{$\begin{array}{l}\text { Core-Section } \\
\text { (level in cm) }\end{array}$} & \multirow{2}{*}{$\begin{array}{l}\text { Corrected } \\
\text { sub-bottom } \\
\text { depth }(m)\end{array}$} & $\begin{array}{l}\text { Planktonic } \\
\text { foraminifers }\end{array}$ & $\begin{array}{l}\text { Benthic } \\
\text { foraminifers }\end{array}$ & \multirow{2}{*}{$\begin{array}{c}\mathrm{CaCO}_{3} \\
(\%)\end{array}$} & Texture $(\%)$ \\
\hline & & $\delta^{18} \mathrm{O}$ & $\delta^{18} \mathrm{O}$ & & Clay Sand \\
\hline
\end{tabular}

Hole 594

\begin{tabular}{|c|c|c|c|c|c|c|c|c|c|}
\hline $1-1,30$ & 0.31 & 1.61 & 1.44 & 3.04 & 0.50 & - & 63.7 & 22.7 & 13.6 \\
\hline $1-1,52$ & 0.53 & 1.48 & 1.21 & 2.77 & 0.44 & 40.2 & 67.0 & 19.6 & 13.4 \\
\hline $1-1,80$ & 0.81 & 1.72 & 0.77 & 3.22 & 0.16 & 41.8 & 71.3 & 22.0 & 6.7 \\
\hline $1-1,105$ & 1.06 & 2.06 & 0.91 & 3.30 & 0.22 & 42.6 & 69.4 & 23.7 & 6.9 \\
\hline $1-1,130$ & 1.31 & 2.92 & 1.04 & 3.86 & 0.31 & 37.9 & 67.8 & 23.7 & 8.5 \\
\hline $1-2,30$ & 1.81 & 3.02 & 0.63 & 4.65 & 0.12 & 6.6 & 80.2 & 16.1 & 3.7 \\
\hline $1-2,52$ & 2.03 & 3.02 & 0.02 & 4.75 & 0.09 & 6.8 & 83.3 & 15.2 & 1.5 \\
\hline $1-2,80$ & 2.31 & 3.12 & -0.34 & 4.80 & 0.01 & 7.8 & 74.3 & 23.9 & 1.8 \\
\hline $1-2,105$ & 2.56 & 3.32 & 0.10 & 4.68 & 0.05 & 11.3 & 78.1 & 17.8 & 4.1 \\
\hline $1-2,130$ & 2.81 & 2.73 & 0.16 & 4.71 & 0.24 & 7.1 & 81.8 & 15.5 & 2.7 \\
\hline $1-3,30$ & 3.31 & 3.27 & 0.99 & 5.10 & 0.26 & 7.1 & 80.3 & 17.7 & 2.0 \\
\hline $1-3,52$ & 3.53 & 3.12 & 0.01 & 4.50 & 0.45 & 3.2 & 78.3 & 20.2 & 1.5 \\
\hline $1-3,80$ & 3.81 & 3.65 & 1.15 & 4.28 & 0.23 & 9.5 & 83.6 & 13.0 & 3.4 \\
\hline $1-3,105$ & 4.06 & 3.37 & 1.40 & 4.44 & 0.65 & 14.3 & 81.6 & 15.5 & 2.9 \\
\hline $1-3,130$ & 4.31 & 2.93 & 0.70 & 4.25 & 1.41 & 6.2 & 80.2 & 18.3 & 1.5 \\
\hline $1-4,30$ & 4.81 & 3.31 & 1.32 & 4.12 & 0.33 & 9.9 & 78.3 & 19.2 & 2.5 \\
\hline $1-4,52$ & 5.03 & 2.89 & 0.46 & 4.25 & 0.51 & 10.3 & 80.3 & 15.6 & 4.1 \\
\hline $1-4,80$ & 5.31 & 3.45 & 1.48 & 4.47 & 0.69 & 8.6 & 80.0 & 17.0 & 3.0 \\
\hline $1-4,105$ & 5.56 & 2.42 & 1.07 & 4.04 & 0.55 & 22.3 & 78.0 & 17.9 & 4.1 \\
\hline $2-1,30$ & 6.21 & 2.61 & 0.82 & 3.92 & 0.37 & - & 65.8 & 25.4 & 8.8 \\
\hline $2-1,52$ & 6.43 & 2.54 & 1.45 & 3.97 & 0.67 & 21.8 & 75.0 & 19.9 & 5.1 \\
\hline $2-1,80$ & 6.71 & 2.52 & 0.31 & 4.11 & 0.48 & 14.5 & 76.9 & 20.2 & 2.9 \\
\hline $2-1,105$ & 6.96 & 2.62 & 0.85 & $3.77^{*}$ & $0.59^{*}$ & 26.0 & 71.1 & 21.1 & 7.8 \\
\hline $2-1,130$ & 7.21 & 2.70 & 1.38 & 3.75 & 0.40 & 29.4 & 70.8 & 22.5 & 6.7 \\
\hline $2-2,30$ & 7.71 & 2.50 & 1.17 & 3.84 & 0.34 & 31.6 & 66.4 & 26.5 & 7.1 \\
\hline $2-2,52$ & 7.93 & 2.93 & 1.18 & 3.90 & 0.30 & 35.9 & 65.7 & 27.9 & 6.4 \\
\hline $2-2,80$ & 8.21 & $2.88,2.97$ & $1.05,1.06$ & 4.05 & 0.15 & 12.8 & 74.2 & 22.5 & 3.3 \\
\hline $2-2,105$ & 8.46 & $2.63,2.79$ & $0.98,0.76$ & 4.04 & 0.30 & 7.2 & 75.1 & 23.0 & 1.9 \\
\hline $2-2,130$ & 8.71 & $2.95,2.94$ & $0.80,0.65$ & 3.91 & -0.11 & 10.3 & 74.4 & 24.2 & 1.4 \\
\hline $2-3,30$ & 9.21 & 3.28 & 0.85 & 4.01 & 0.13 & 7.0 & 74.2 & 25.0 & 0.8 \\
\hline $2-3,52$ & 9.43 & 3.23 & 1.00 & 4.44 & 0.20 & 7.9 & 78.1 & 20.8 & 1.1 \\
\hline $2-3,80$ & 9.71 & 3.29 & 0.52 & 4.30 & 0.16 & 10.0 & 78.3 & 20.5 & 1.2 \\
\hline $2-3,105$ & 9.96 & $3.25,3.08$ & $0.11,0.97$ & 4.49 & 0.14 & 11.2 & 78.4 & 19.9 & 1.7 \\
\hline $2-3,130$ & 10.21 & $2.97,3.01$ & $0.13,0.88$ & 4.27 & 0.31 & 15.4 & 75.0 & 23.4 & 1.6 \\
\hline $2-4,30$ & 10.71 & $2.97,2.99$ & $1.38,1.70$ & 4.03 & 0.29 & 35.6 & 67.6 & 26.2 & 6.2 \\
\hline $2-4,52$ & 10.93 & $2.63,2.74$ & $1.20,1.79$ & 3.33 & 0.40 & 48.0 & 52.5 & 39.9 & 7.6 \\
\hline $2-4,80$ & 11.21 & 2.77 & 1.79 & 3.76 & 0.44 & 42.2 & 52.9 & 35.4 & 11.7 \\
\hline $2-4,105$ & 11.46 & 2.78 & 1.75 & 3.50 & 0.27 & 45.1 & 59.2 & 36.6 & 4.2 \\
\hline $2-4,130$ & 11.71 & 2.40 & 1.26 & 3.69 & 0.20 & 47.6 & 58.3 & 33.2 & 8.5 \\
\hline $2-5,30$ & 12.21 & 2.52 & 1.51 & 3.53 & 0.39 & 55.3 & 60.8 & 34.2 & 5.0 \\
\hline $2-5,52$ & 12.43 & 2.69 & 1.85 & 3.96 & 0.65 & 53.6 & 60.5 & 33.2 & 6.3 \\
\hline $2-5,80$ & 12.71 & 2.58 & 1.75 & 3.77 & 0.61 & 28.0 & 61.7 & 24.4 & 13.9 \\
\hline $2-5,105$ & 12.96 & 2.68 & 1.68 & 3.87 & 0.39 & 38.3 & 53.9 & 34.7 & 11.4 \\
\hline $2-5,130$ & 13.21 & 2.26 & 1.69 & 3.81 & 0.47 & 21.5 & 61.7 & 32.5 & 5.8 \\
\hline $2-6,30$ & 13.71 & 2.24 & 1.67 & 3.38 & 0.57 & 51.5 & 49.8 & 38.7 & 11.5 \\
\hline $2-6,52$ & 13.93 & 2.55 & 1.62 & 3.39 & 0.29 & 54.4 & 49.8 & 35.3 & 14.9 \\
\hline $2-6,80$ & 14.21 & 2.39 & 1.26 & 2.92 & -0.13 & 45.0 & 46.1 & 30.1 & 23.8 \\
\hline $2-6,105$ & 14.46 & 2.58 & 1.41 & 3.08 & 0.25 & 44.6 & 63.4 & 27.2 & 9.4 \\
\hline $3-1,30$ & 15.81 & 2.54 & 1.44 & 3.21 & -0.43 & 22.1 & 62.3 & 11.4 & 26.3 \\
\hline $3-1,52$ & 16.03 & 1.35 & 0.71 & $2.83^{*}$ & $-0.08^{*}$ & 55.4 & 58.9 & 24.6 & 16.5 \\
\hline $3-1,80$ & 16.30 & 1.59 & 0.49 & 2.94 & 0.12 & 60.7 & 63.7 & 30.6 & 5.7 \\
\hline $3-1,105$ & 16.56 & 2.97 & 0.85 & 4.00 & 0.10 & 45.2 & 69.7 & 23.0 & 7.3 \\
\hline $3-1,130$ & 16.81 & 3.20 & 0.88 & 4.27 & 0.25 & 28.1 & 78.8 & 17.5 & 3.7 \\
\hline $3-2,30$ & 17.31 & $3.17,3.16$ & $1.01,0.93$ & 3.96 & 0.21 & 4.5 & 73.5 & 22.2 & 4.3 \\
\hline $3-2,52$ & 17.53 & $2.35,2.39$ & $0.85,1.08$ & $3.80^{*}$ & $0.50^{*}$ & 6.1 & 80.3 & 16.8 & 2.9 \\
\hline $3-2,80$ & 17.80 & $2.71,2.47$ & $1.02,0.91$ & 3.97 & 0.07 & 8.9 & 75.7 & 21.1 & 3.2 \\
\hline $3-2,105$ & 18.06 & $3.22,3.03$ & $0.70,1.17$ & 3.63 & -0.16 & 20.5 & 76.0 & 17.7 & 6.3 \\
\hline $3-2,130$ & 18.31 & 2.97 & 0.68 & 4.35 & -0.07 & 19.8 & 75.5 & 18.6 & 5.9 \\
\hline $3-3,30$ & 18.81 & $3.26,3.31$ & $0.69,0.55$ & 4.43 & -0.15 & 4.7 & 81.8 & 16.0 & 2.2 \\
\hline $3-3,52$ & 19.03 & $3.20,2.89$ & $0.72,0.80$ & 4.28 & 0.33 & 20.5 & 75.1 & 19.4 & 5.5 \\
\hline $3-3,80$ & 19.31 & $3.04,3.03$ & $0.80,0.93$ & 4.40 & 0.15 & 12.2 & 74.9 & 21.7 & 3.4 \\
\hline $3-3,105$ & 19.80 & $2.79,2.92$ & $0.78,0.95$ & - & - & 5.9 & 79.3 & 19.0 & 1.7 \\
\hline $3-3,130$ & 19.81 & $3.51,3.01$ & $0.24,0.11$ & - & - & 4.0 & 80.4 & 17.6 & 2.0 \\
\hline $3-4,30$ & 20.31 & $3.24,3.45$ & $1.10,0.86$ & 4.00 & -0.09 & 30.7 & 73.2 & 18.2 & 8.6 \\
\hline $3-4,52$ & 20.53 & $2.68,3.23$ & $0.51,1.09$ & 3.83 & 0.18 & 15.7 & 79.5 & 14.3 & 6.2 \\
\hline $3-4,80$ & 20.80 & $3.02,2.94$ & $0.90,1.01$ & 4.08 & 0.11 & 8.2 & 77.4 & 18.8 & 3.8 \\
\hline $3-4,105$ & 21.06 & $3.47,3.13$ & $0.97,1.11$ & 3.91 & 0.01 & 9.8 & 77.2 & 14.9 & 7.9 \\
\hline $3-4,140$ & 21.40 & $2.68,3.09$ & $1.20,0.85$ & 3.68 & 0.13 & 22.7 & 73.1 & 15.5 & 11.4 \\
\hline $3-5,30$ & 21.81 & $2.35,2.27$ & $1.20,1.09$ & 4.63 & -0.95 & 4.8 & 76.0 & 22.7 & 1.3 \\
\hline $3-5,52$ & 22.03 & $3.28,3.10$ & $1.08,1.10$ & - & - & 8.2 & 78.0 & 13.2 & 8.8 \\
\hline $3-5,80$ & 22.30 & $3.09,3.21$ & $0.83,0.99$ & - & - & 6.0 & 80.0 & 17.3 & 2.7 \\
\hline $3-5,105$ & 22.56 & $3.47,2.88$ & $0.85,0.84$ & 3.62 & 0.10 & 19.6 & 77.5 & 14.8 & 7.7 \\
\hline
\end{tabular}


Table 2. (Continued).

\begin{tabular}{|c|c|c|c|c|c|c|c|c|c|}
\hline \multirow{2}{*}{$\begin{array}{l}\text { Core-Section } \\
\text { (level in } \mathrm{cm} \text { ) }\end{array}$} & \multirow{2}{*}{$\begin{array}{l}\text { Corrected } \\
\text { sub-bottom } \\
\text { depth (m) }\end{array}$} & \multicolumn{2}{|c|}{$\begin{array}{l}\text { Planktonic } \\
\text { foraminifers }\end{array}$} & \multicolumn{2}{|c|}{$\begin{array}{c}\text { Benthic } \\
\text { foraminifers }\end{array}$} & \multirow{2}{*}{$\begin{array}{c}\mathrm{CaCO}_{3} \\
(\%)\end{array}$} & \multicolumn{3}{|c|}{ Texture $(\%)$} \\
\hline & & $\delta^{18} \mathrm{O}$ & ${ }^{13} \mathrm{C}$ & $\delta^{18} \mathrm{O}$ & $\delta^{13} \mathrm{C}$ & & Silt & Clay & Sand \\
\hline Hole 594 (Con & & & & & & & 7 & & \\
\hline $3-5,130$ & 22.81 & $2.99,3.22$ & $0.83,1.02$ & 4.25 & -0.39 & 5.2 & 77.0 & 18.0 & 5.0 \\
\hline $3-6,30$ & 23.31 & 1.23 & 0.68 & $2.96^{*}$ & $-0.60^{*}$ & 56.8 & 68.3 & 16.8 & 14.9 \\
\hline $3-6,52$ & 23.53 & 2.03 & 0.83 & $3.30^{*}$ & $0.49^{*}$ & 40.5 & 44.4 & 11.3 & 44.3 \\
\hline $3-6,80$ & 23.80 & 1.48 & 0.46 & $3.13^{*}$ & $0.28^{*}$ & 44.9 & 60.4 & 15.0 & 24.6 \\
\hline $3-6,105$ & 24.06 & 2.44 & 1.07 & $3.21^{*}$ & $0.75^{*}$ & 48.7 & 58.6 & 24.4 & 17.0 \\
\hline $3-6,130$ & 24.31 & 2.19 & 0.95 & $3.29^{*}$ & $0.09^{*}$ & - & 60.3 & 15.4 & 24.3 \\
\hline $4-1,30$ & 25.41 & 2.27 & 1.08 & - & - & 34.1 & 66.2 & 23.8 & 10.0 \\
\hline $4-1,52$ & 25.63 & 2.11 & 0.97 & - & - & 47.1 & 56.2 & 17.8 & 26.0 \\
\hline $4-1,80$ & 25.91 & 2.27 & 0.90 & $2.82^{*}$ & $0.21^{*}$ & 50.0 & 61.7 & 23.0 & 15.3 \\
\hline $4-1,105$ & 26.16 & 1.98 & 0.75 & $2.83^{*}$ & $-0.21^{*}$ & 53.7 & 68.2 & 23.6 & 8.2 \\
\hline $4-1,130$ & 26.41 & 1.61 & 0.66 & 2.70 & 0.25 & 56.8 & 68.0 & 27.6 & 4.4 \\
\hline $4-2,30$ & 26.91 & 2.53 & 0.74 & 3.75 & 0.13 & 21.9 & 78.2 & 15.8 & 6.0 \\
\hline $4-2,52$ & 27.13 & 2.71 & 0.76 & 3.76 & 0.23 & 31.1 & 72.7 & 19.3 & 8.0 \\
\hline $4-2,80$ & 27.41 & 2.38 & 0.69 & - & - & 8.7 & 81.6 & 16.3 & 2.1 \\
\hline $4-2,105$ & 27.66 & $2.61,2.72$ & $0.94,0.71$ & 3.55 & -0.01 & 12.1 & 79.6 & 17.1 & 3.3 \\
\hline $4-2,130$ & 27.91 & $3.27,2.86$ & $0.45,0.30$ & 3.77 & -0.25 & 12.9 & 78.3 & 15.7 & 6.0 \\
\hline $4-3,30$ & 28.41 & $2.67,2.45$ & $0.70,0.58$ & 4.04 & 0.24 & 9.9 & 79.6 & 16.6 & 3.8 \\
\hline $4-3,52$ & 28.63 & $3.17,3.09$ & $1.02,0.84$ & 4.08 & 0.14 & 24.6 & 71.3 & 20.0 & 8.7 \\
\hline $4-3,80$ & 28.91 & $3.38,3.49$ & $0.99,0.82$ & 3.85 & 0.05 & 20.3 & 74.3 & 19.1 & 6.6 \\
\hline $4-3,130$ & 29.41 & $3.02,2.71$ & $0.89,0.41$ & 3.98 & -0.01 & 8.5 & 79.3 & 18.2 & 2.5 \\
\hline $4-4,30$ & 29.91 & $2.65,2.38$ & $0.70,0.31$ & 3.72 & -0.05 & 4.2 & 79.0 & 19.0 & 2.0 \\
\hline $4-4,52$ & 30.13 & $2.71,2.42$ & $0.57,0.60$ & - & - & 2.9 & 77.2 & 20.5 & 2.3 \\
\hline $4-4,80$ & 30.41 & $2.84,2.87$ & $0.46,0.93$ & 4.43 & 0.14 & 2.6 & 82.5 & 15.3 & 2.2 \\
\hline $4-4,105$ & 30.66 & 2.95 & 1.05 & - & - & 5.1 & 80.2 & 17.0 & 2.8 \\
\hline $4-4,130$ & 30.91 & 3.19 & 0.58 & 4.44 & 0.18 & 4.4 & 80.9 & 17.4 & 1.7 \\
\hline $4-5,30$ & 31.41 & $2.49,3.07$ & $1.63,1.52$ & 4.03 & 0.52 & 30.6 & 64.9 & 19.4 & 15.7 \\
\hline $4-5,52$ & 31.63 & $2.60,2.72$ & $1.58,1.44$ & - & - & 48.6 & 58.0 & 25.9 & 16.1 \\
\hline $4-5,80$ & 31.91 & $2.68,2.79$ & $1.46,1.75$ & - & - & 46.0 & 64.4 & 28.4 & 7.2 \\
\hline $4-5,105$ & 32.16 & $2.56,2.56$ & $1.51,1.49$ & $3.52^{*}$ & $-0.47^{*}$ & 31.4 & 64,7 & 22.2 & 13.1 \\
\hline $4-5,130$ & 32.41 & 2.68 & 1.34 & 3.18 & -0.07 & 29.0 & 67.9 & 23.5 & 8.6 \\
\hline $4-6,30$ & 32.91 & 2.27 & 1.77 & - & - & 49.0 & 61.2 & 29.7 & 9.1 \\
\hline $4-6,52$ & 33.13 & $2.32,2.82$ & $1.67,1.61$ & - & - & 42.5 & 56.8 & 27.1 & 16.1 \\
\hline $4-6,80$ & 33.41 & $2.26,2.54$ & $1.63,1.34$ & - & - & 43.8 & 54.9 & 24.2 & 20.9 \\
\hline $4-6,105$ & 33.66 & $1.95,2.10$ & $1.63,1.12$ & - & - & 45.8 & 57.1 & 24.4 & 18.5 \\
\hline $5-1,52$ & 35.23 & $2.30,2.77$ & $1.06,1.26$ & - & - & 8.1 & 83.7 & 13.4 & 2.9 \\
\hline $5-1,80$ & 35.51 & $2.61,2.52$ & $1.03,1.08$ & - & - & 12.8 & 81.1 & 15.9 & 3.0 \\
\hline $5-1,105$ & 35.76 & $2.23,2.63$ & $1.48,1.27$ & 4.30 & 0.20 & 15.5 & 76.4 & 21.9 & 1.7 \\
\hline $5-1,132$ & 36.03 & 3.48 & 1.06 & - & - & 9.3 & 82.2 & 15.8 & 2.0 \\
\hline $5-2,30$ & 36.51 & 2.31 & 1.28 & - & - & 2.7 & 54.3 & 7.6 & 38.1 \\
\hline $5-2,52$ & 36.73 & 3.44 & 0.66 & - & - & 19.6 & 79.4 & 18.7 & 1.9 \\
\hline $5-2,80$ & 37.01 & 3.04 & 1.29 & 4.25 & -0.14 & 22.6 & 73.3 & 25.4 & 1.3 \\
\hline $5-2,105$ & 37.26 & 3.17 & 1.96 & 4.42 & 0.12 & 38.3 & - & - & 3.2 \\
\hline $5-2,132$ & 37.53 & 3.24 & 1.38 & 4.10 & 0.09 & 39.7 & 61.4 & 35.2 & 3.4 \\
\hline $5-3,30$ & 38.01 & 3.31 & 1.48 & $3.57^{*}$ & $-0.05^{*}$ & 32.3 & 70.4 & 26.3 & 3.3 \\
\hline $5-3,52$ & 38.23 & 2.94 & 1.44 & $4.08^{*}$ & $0.35^{*}$ & 22.8 & 62.1 & 34.7 & 3.2 \\
\hline $5-3,80$ & 38.51 & 2.42 & 1.14 & - & - & 16.0 & 71.6 & 27.4 & 1.0 \\
\hline $5-3,105$ & 38.76 & 2.93 & 1.30 & 4.26 & 0.14 & 18.4 & 76.4 & 21.4 & 2.2 \\
\hline $5-3,132$ & 39.03 & 3.41 & 1.23 & - & - & 16.0 & 79.0 & 19.6 & 1.4 \\
\hline $5-4,30$ & 39.51 & 2.91 & 1.62 & 4.18 & 0.27 & 17.6 & 74.7 & 23.2 & 2.1 \\
\hline $5-4,52$ & 39.73 & 2.92 & 1.79 & - & - & 20.5 & 78.0 & 20.5 & 1.5 \\
\hline $5-4,80$ & 40.01 & 3.02 & 1.63 & - & - & 23.4 & 72.1 & 26.1 & 1.8 \\
\hline $5-4,132$ & 40.53 & 2.73 & 1.63 & - & - & 45.5 & 57.8 & 39.7 & 2.5 \\
\hline $5-5,30$ & 41.01 & 2.93 & 1.71 & 4.07 & 0.23 & 24.3 & 70.7 & 27.5 & 1.8 \\
\hline $5-5,52$ & 41.23 & 2.72 & 1.94 & - & - & 26.4 & 70.8 & 25.2 & 4.0 \\
\hline $5-5,80$ & 41.51 & 2.70 & 1.56 & 4.20 & -0.12 & 35.0 & 69.7 & 22.6 & 7.7 \\
\hline $5-5,105$ & 41.76 & 2.45 & 1.58 & 3.92 & -0.08 & 41.3 & 69.9 & 21.4 & 8.7 \\
\hline $5-5,132$ & 42.03 & 2.49 & 1.81 & 3.68 & 0.08 & 52.3 & 70.0 & 27.6 & 2.4 \\
\hline $5-6,30$ & 42.51 & 2.94 & 1.71 & 3.93 & 0.07 & - & 62.0 & 31.2 & 6.8 \\
\hline $5-6,52$ & 42.73 & 2.89 & 1.67 & 3.62 & 0.18 & 57.9 & 59.9 & 34.1 & 6.0 \\
\hline $5-6,80$ & 43.01 & 2.87 & 1.68 & - & - & 60.4 & 43.2 & 46.5 & 10.3 \\
\hline $5-6,105$ & 43.26 & 2.61 & 1.65 & $3.95^{*}$ & $0.26^{*}$ & 68.0 & 55.5 & 38.5 & 6.0 \\
\hline $5-6,132$ & 43.53 & 2.52 & 1.79 & 3.65 & 0.30 & 60.2 & 47.3 & 45.0 & 7.7 \\
\hline $5-7,30$ & 44.01 & 2.68 & 1.81 & 3.65 & 0.27 & 63.3 & - & - & 3.9 \\
\hline $9-1,52$ & 73.63 & 2.03 & 0.40 & 4.35 & 0.13 & 41.1 & 66.8 & 28.8 & 4.4 \\
\hline $9-1,80$ & 73.91 & 2.23 & 0.43 & 3.84 & 0.15 & 54.9 & 56.5 & 39.2 & 4.3 \\
\hline $9-1,105$ & 74.16 & 1.83 & 0.42 & 3.62 & 0.37 & 60.4 & 52.7 & 36.9 & 10.4 \\
\hline $9-1,130$ & 74.41 & 1.50 & 0.24 & 3.39 & 0.30 & 56.9 & 53.3 & 39.5 & 7.2 \\
\hline $9-2,30$ & 74.91 & 1.78 & 0.54 & 3.39 & 0.44 & 61.7 & 49.0 & 40.7 & 10.3 \\
\hline $9-2,52$ & 75.13 & 1.42 & 0.38 & 3.08 & 0.72 & 68.4 & 58.9 & 39.2 & 1.9 \\
\hline $9-2,80$ & 75.41 & 1.76 & 0.24 & 3.61 & 0.42 & 70.1 & 49.1 & 39.7 & 11.2 \\
\hline $9-2,105$ & 75.66 & 2.08 & 0.01 & 3.49 & 0.03 & 47.2 & 71.6 & 19.8 & 8.6 \\
\hline $9-2,130$ & 75.91 & 2.45 & 0.16 & 3.77 & -0.14 & 41.8 & 69.9 & 23.6 & 6.5 \\
\hline $9-3,30$ & 76.41 & 2.14 & - & - & - & 4.8 & 84.0 & 15.0 & 1.0 \\
\hline $9-3,52$ & 76.63 & 3.57 & - & - & - & 4.0 & 72.6 & 26.2 & 1.2 \\
\hline
\end{tabular}


Table 2. (Continued).

\begin{tabular}{|c|c|c|c|c|c|c|c|c|c|}
\hline \multirow{2}{*}{$\begin{array}{l}\text { Core-Section } \\
\text { (level in } \mathrm{cm} \text { ) }\end{array}$} & \multirow{2}{*}{$\begin{array}{l}\text { Corrected } \\
\text { sub-bottom } \\
\text { depth }(m)\end{array}$} & \multicolumn{2}{|c|}{$\begin{array}{l}\text { Planktonic } \\
\text { foraminifers }\end{array}$} & \multicolumn{2}{|c|}{$\begin{array}{l}\text { Benthic } \\
\text { foraminifers }\end{array}$} & \multirow{2}{*}{$\begin{array}{c}\mathrm{CaCO}_{3} \\
(\%)\end{array}$} & \multicolumn{3}{|c|}{ Texture $(\%)$} \\
\hline & & ${ }^{18} \mathrm{O}$ & ${ }^{13} \mathrm{C}$ & $\delta^{18} \mathrm{O}$ & ${ }^{13} \mathrm{C}$ & & Silt & Clay & Sand \\
\hline
\end{tabular}

Hole 594 (Cont).

\begin{tabular}{|c|c|c|c|c|c|c|c|c|}
\hline $9-3,80$ & 76.91 & 3.18 & - & - & - & 10.6 & 84.7 & 13.8 \\
\hline $9-3,105$ & 77.16 & 3.37 & 0.71 & 3.61 & 0.02 & 53.3 & 73.4 & 17.6 \\
\hline $9-3,130$ & 77.41 & 2.68 & 0.28 & 3.22 & -0.16 & 49.0 & 73.6 & 23.4 \\
\hline $9-4,30$ & 77.91 & 2.71 & 0.16 & 3.85 & -0.04 & 26.6 & 77.9 & 13.3 \\
\hline $9-4,80$ & 78.41 & 3.01 & 0.13 & 4.63 & 0.17 & 9.5 & 83.7 & 12.4 \\
\hline $9-4,105$ & 78.66 & 3.47 & 0.67 & - & - & 7.7 & 83.3 & 15.2 \\
\hline $9-4,130$ & 78.91 & 3.89 & 0.34 & 4.39 & -0.41 & 8.9 & 83.9 & 14.5 \\
\hline $9-5,30$ & 79.41 & 3.03 & 0.56 & - & - & 9.1 & 81.7 & 14.7 \\
\hline $9-5,52$ & 79.63 & 3.65 & 0.72 & 4.30 & -0.28 & 13.1 & 84.7 & 12.3 \\
\hline $9-5,80$ & 79.91 & 3.22 & 0.86 & - & - & 15.2 & 83.0 & 13.9 \\
\hline $9-5,105$ & 80.16 & 2.86 & 0.74 & 4.23 & -0.29 & 15.9 & 83.2 & 14.5 \\
\hline $9-5,130$ & 80.41 & 2.50 & 0.31 & 3.53 & 0.26 & 42.8 & 75.6 & 15.9 \\
\hline $9-6,30$ & 80.91 & 2.11 & 0.38 & 3.29 & 0.26 & 56.6 & 61.8 & 24.0 \\
\hline $9-6,52$ & 81.13 & 2.25 & 0.27 & 3.82 & -0.10 & 62.3 & 51.7 & 29.3 \\
\hline $9-6,80$ & 81.41 & 2.14 & 0.53 & 3.00 & -0.26 & 68.7 & 45.2 & 41.0 \\
\hline $9-6,105$ & 81.66 & 1.63 & 0.74 & 2.94 & 0.06 & 70.0 & 50.4 & 33.0 \\
\hline $9-6,130$ & 81.91 & 1.59 & 0.47 & 3.54 & 0.30 & 74.9 & 52.3 & 43.0 \\
\hline $11-1,52$ & 92.83 & 2.63 & 0.54 & - & - & 2.0 & 84.0 & 14.6 \\
\hline $11-1,80$ & 93.11 & - & - & - & - & 3.3 & 82.5 & 15.7 \\
\hline $11-1,105$ & 93.36 & 2.23 & 0.97 & 3.81 & -0.23 & 16.1 & 78.4 & 14.7 \\
\hline $11-1,130$ & 93.61 & 2.63 & 1.23 & 3.67 & -0.18 & 46.7 & 68.9 & 19.7 \\
\hline $11-2,30$ & 94.11 & 1.91 & 0.62 & 3.67 & 0.25 & 50.6 & 58.6 & 18.7 \\
\hline $11-2,52$ & 94.33 & 2.64 & 0.82 & 3.72 & 0.07 & 16.6 & 79.5 & 17.3 \\
\hline $11-2,80$ & 94.61 & 2.50 & 1.34 & 3.09 & 0.60 & 45.5 & 73.2 & 14.6 \\
\hline $11-2,105$ & 94.86 & 2.59 & 1.21 & 2.86 & 0.25 & 36.2 & 72.0 & 12.3 \\
\hline $11-2,130$ & 95.11 & 2.62 & 1.26 & 3.23 & 0.39 & 40.1 & 67.5 & 12.9 \\
\hline $11-3,30$ & 95.61 & 2.48 & 1.23 & 3.34 & 0.60 & 42.4 & 72.6 & 14.8 \\
\hline $11-3,52$ & 95.83 & 2.42 & 0.71 & $3.29^{\circ}$ & $-0.12^{*}$ & 43.9 & 76.1 & 13.7 \\
\hline $11-3,80$ & 96.11 & 2.59 & 1.04 & 3.39 & -0.43 & 21.8 & 80.8 & 13.8 \\
\hline $11-3,105$ & 96.36 & 2.62 & 0.46 & 3.62 & -0.52 & 19.4 & 79.6 & 16.2 \\
\hline $11-3,130$ & 96.61 & 2.19 & -0.47 & 3.84 & -0.32 & 12.3 & 83.6 & 14.8 \\
\hline $11-4,30$ & 97.11 & 2.61 & 0.59 & 3.94 & -0.02 & 13.4 & 84.9 & 12.4 \\
\hline $11-4,52$ & 97.33 & 2.70 & 0.47 & 3.82 & -0.36 & 38.2 & 83.7 & 13.6 \\
\hline $11-4,80$ & 97.61 & 2.45 & 0.73 & 3.94 & -0.09 & 36.0 & 75.9 & 18.8 \\
\hline $11-4,105$ & 97.86 & 1.98 & 1.19 & 3.40 & 0.23 & 64.0 & 64.9 & 26.2 \\
\hline $11-4,130$ & 98.11 & 2.03 & 0.99 & 3.15 & 0.10 & 76.9 & 62.1 & 30.3 \\
\hline $11-5,30$ & 98.61 & 1.43 & 0.40 & - & - & 69.2 & 53.0 & 38.2 \\
\hline $11-5,52$ & 98.83 & 2.18 & 1.16 & 3.51 & -0.02 & 59.8 & 69.7 & 22.2 \\
\hline $11-5,80$ & 99.11 & 2.63 & 1.08 & 3.52 & 0.19 & 38.3 & 69.5 & 24.1 \\
\hline $11-5,105$ & 99.36 & 2.37 & 0.72 & 3.46 & -0.18 & 27.0 & 69.8 & 23.8 \\
\hline $11-5,130$ & 99.61 & 3.27 & 1.18 & 3.69 & 0.52 & 39.5 & 66.6 & 18.4 \\
\hline $11-6,30$ & 100.11 & 2.72 & 0.89 & 3.79 & -0.11 & 22.4 & 78.3 & 11.2 \\
\hline $11-6,52$ & 100.33 & 2.06 & 1.01 & 3.99 & -0.24 & 17.2 & 76.6 & 14.2 \\
\hline $11-6,80$ & 100.61 & 2.46 & 1.04 & 3.95 & -0.37 & 12.6 & 83.8 & 13.3 \\
\hline $11-6,105$ & 100.86 & 2.26 & 1.22 & 3.65 & 0.25 & 29.9 & 73.5 & 11.8 \\
\hline $11-6,130$ & 101.11 & 1.98 & 1.31 & - & - & 54.0 & 63.4 & 19.6 \\
\hline $11-7,30$ & 101.61 & 1.42 & 0.83 & - & - & 54.5 & 56.6 & 17.4 \\
\hline
\end{tabular}

Hole 594A

\begin{tabular}{llllllllll}
$1-3,30$ & 44.61 & 2.11 & 1.88 & 3.22 & 0.43 & 77.4 & 53.8 & 40.8 & 5.4 \\
$1-3,52$ & 44.83 & 2.11 & 1.37 & 2.77 & 0.38 & 81.9 & 47.1 & 48.1 & 4.8 \\
$1-3,80$ & 45.11 & 0.96 & 0.54 & 2.77 & 0.79 & 76.1 & 49.0 & 44.1 & 6.9 \\
$1-3,105$ & 45.36 & 1.72 & 1.38 & 2.86 & 0.84 & 75.3 & 56.7 & 37.6 & 5.7 \\
$1-3,130$ & 45.61 & 1.94 & 1.67 & 3.02 & 1.03 & 76.6 & 55.5 & 39.7 & 4.8 \\
$1-4,30$ & 46.11 & 1.73 & 0.98 & - & - & 77.6 & 51.6 & 44.3 & 4.1 \\
$1-4,52$ & 46.33 & 2.27 & 1.01 & - & - & 69.1 & 55.7 & 37.5 & 6.8 \\
$1-4,80$ & 46.61 & 2.68 & 1.19 & - & - & 60.1 & 64.3 & 28.5 & 7.2 \\
$1-4,105$ & 46.86 & 2.26 & 0.57 & - & - & 58.3 & 61.2 & 32.9 & 5.9 \\
$1-4,130$ & 47.11 & 3.32 & 1.09 & $4.28 *$ & $0.13 *$ & 45.9 & 62.8 & 34.6 & 2.6 \\
$1-5,30$ & 47.61 & 3.30 & 0.86 & - & - & 19.5 & 79.3 & 17.8 & 2.9 \\
$1-5,52$ & 47.83 & 3.06 & -0.01 & - & - & 16.1 & 82.3 & 16.2 & 1.5 \\
$1-5,80$ & 48.11 & 3.48 & 0.85 & - & - & 7.7 & - & - & -5.7 \\
$1-5,105$ & 48.36 & 3.45 & 0.66 & - & - & 6.5 & 85.1 & 13.2 & 1.7 \\
$1-5,130$ & 48.61 & 2.90 & 0.70 & - & - & 5.9 & 82.0 & 16.9 & 1.1 \\
$1-6,30$ & 49.11 & 3.23 & 0.50 & - & - & 2.5 & 80.8 & 18.2 & 1.0 \\
$1-6,52$ & 49.33 & 3.07 & 0.39 & - & - & 11.3 & 82.3 & 16.3 & 1.4 \\
$1-6,80$ & 49.61 & 3.60 & 0.69 & 4.91 & -0.14 & 5.8 & 81.3 & 17.9 & 0.8 \\
$1-6,105$ & 49.86 & 3.13 & 0.61 & 4.93 & -0.05 & 10.5 & 77.9 & 20.0 & 2.1 \\
$1-7,30$ & 50.30 & 2.93 & 0.67 & - & - & - & 84.3 & 13.1 & 2.6 \\
$1-7,52$ & 50.61 & 2.96 & 0.54 & - & - & 4.8 & 83.5 & 15.3 & 1.2 \\
$2-1,30$ & 51.21 & 2.83 & 0.99 & - & - & 14.3 & 77.9 & 20.3 & 1.8 \\
$2-1,52$ & 51.43 & 3.37 & 0.91 & 5.10 & -0.04 & 22.6 & 79.9 & 17.4 & 2.7 \\
$2-1,80$ & 51.71 & 3.03 & 0.34 & 4.46 & 0.01 & 11.5 & 82.3 & 16.5 & 1.2 \\
\hline
\end{tabular}


Table 2. (Continued).

\begin{tabular}{|c|c|c|c|c|c|c|c|c|c|}
\hline \multirow{2}{*}{$\begin{array}{l}\text { Core-Section } \\
\text { (level in cm) }\end{array}$} & \multirow{2}{*}{$\begin{array}{l}\text { Corrected } \\
\text { sub-bottom } \\
\text { depth }(m)\end{array}$} & \multicolumn{2}{|c|}{$\begin{array}{l}\text { Planktonic } \\
\text { foraminifers }\end{array}$} & \multicolumn{2}{|c|}{$\begin{array}{c}\text { Benthic } \\
\text { foraminifers }\end{array}$} & \multirow{2}{*}{$\begin{array}{c}\mathrm{CaCO}_{3} \\
(\%)\end{array}$} & \multicolumn{3}{|c|}{ Texture $(\%)$} \\
\hline & & ${ }_{\delta}^{18} \mathrm{O}$ & $\delta^{13} \mathrm{C}$ & ${ }_{\delta^{18}} \mathrm{O}$ & ${ }_{\delta^{13} \mathrm{C}}$ & & Silt & Clay & Sand \\
\hline Hole $594 \mathrm{~A}(\mathrm{Cc}$ & nt). & & & & & & & & \\
\hline $2-1,105$ & 51.96 & 3.22 & 0.54 & 4.54 & -0.02 & 15.9 & 78.3 & 19.8 & 1.9 \\
\hline $2-1,130$ & 52.21 & 3.38 & 1.44 & 4.42 & 0.01 & 24.8 & 72.3 & 24.4 & 3.3 \\
\hline $2-2,30$ & 52.71 & 2.94 & 0.37 & 4.72 & 0.16 & 19.4 & - & - & 3.6 \\
\hline $2-2,52$ & 52.93 & 2.78 & 0.82 & 4.30 & -0.02 & 15.0 & 74.9 & 22.4 & 2.7 \\
\hline $2-2,80$ & 53.11 & - & - & 4.44 & 0.16 & 8.8 & 70.8 & 16.4 & 12.8 \\
\hline $2-2,105$ & 53.46 & 2.64 & 0.30 & 4.34 & 0.10 & 7.0 & 82.0 & 16.6 & 1.4 \\
\hline $2-2,130$ & 53.71 & 2.58 & 1.44 & 4.35 & 0.07 & 8.7 & 82.6 & 15.9 & 1.5 \\
\hline $2-3,30$ & 54.21 & 2.67 & 1.37 & 4.13 & 0.10 & 17.3 & 70.4 & 25.7 & 3.9 \\
\hline $2-3,52$ & 54.43 & 2.56 & 1.02 & 4.33 & 0.16 & 12.2 & 77.4 & 20.0 & 2.6 \\
\hline $2-3,80$ & 54.71 & 2.26 & 0.49 & 4.54 & 0.21 & 7.5 & 79.2 & 16.5 & 4.3 \\
\hline $2-3,105$ & 54.96 & 2.74 & 1.47 & 4.16 & 0.26 & 12.8 & 79.3 & 18.7 & 2.0 \\
\hline $2-3,130$ & 55.21 & - & - & 4.31 & 0.21 & 14.0 & 78.7 & 19.0 & 2.3 \\
\hline $2-4,30$ & 55.71 & 2.10 & 1.42 & 3.46 & 0.23 & 54.1 & 62.3 & 32.1 & 5.6 \\
\hline $2-4,52$ & 55.93 & 2.58 & 1.88 & 3.38 & 0.61 & 76.2 & 53.4 & 41.8 & 4.8 \\
\hline $2-4,80$ & 56.21 & 2.40 & 1.73 & 3.08 & 0.94 & 60.3 & 55.8 & 36.1 & 8.1 \\
\hline $2-4,105$ & 56.46 & 2.32 & 1.85 & 3.13 & 0.76 & 70.3 & 55.1 & 37.1 & 7.8 \\
\hline $2-4,130$ & 56.71 & 1.97 & 1.32 & 3.32 & 0.67 & 66.7 & 62.0 & 30.6 & 7.4 \\
\hline $2-5,30$ & 57.21 & 2.31 & 1.46 & 3.46 & 0.50 & 52.8 & 61.5 & 28.0 & 10.5 \\
\hline $2-5,52$ & 57.43 & 2.85 & 1.20 & 3.59 & 0.41 & 46.8 & 66.6 & 27.4 & 6.0 \\
\hline $2-5,80$ & 57.71 & 2.39 & 1.60 & 3.86 & 0.55 & 50.0 & 64.6 & 29.2 & 6.2 \\
\hline $2-5,105$ & 57.96 & 2.12 & 1.52 & 4.21 & 0.52 & 44.0 & 69.6 & 26.0 & 4.4 \\
\hline $2-5,130$ & 58.21 & 2.70 & 2.02 & 3.82 & 0.56 & 52.3 & 46.0 & 34.6 & 19.4 \\
\hline $2-6,30$ & 58.71 & 2.57 & 1.20 & 3.74 & 0.62 & 51.4 & 66.3 & 22.2 & 11.5 \\
\hline $2-6,80$ & 59.21 & 2.41 & 0.74 & 3.62 & 0.35 & 41.5 & 62.1 & 28.9 & 9.0 \\
\hline $2-6,105$ & 59.46 & 2.54 & 0.12 & 4.47 & -0.05 & 23.8 & 77.9 & 18.4 & 3.7 \\
\hline $2-6,130$ & 59.71 & 2.35 & 1.20 & 4.11 & 0.11 & 10.6 & 81.0 & 17.0 & 2.0 \\
\hline $3-1,30$ & 60.81 & 2.28 & 0.51 & - & - & 13.3 & 82.4 & 15.1 & 2.5 \\
\hline $3-1,52$ & 61.03 & 2.42 & 0.75 & 4.06 & 0.47 & 4.6 & 81.3 & 16.5 & 2.2 \\
\hline $3-1,80$ & 61.31 & 3.28 & 1.21 & - & - & 8.6 & 84.0 & 13.7 & 2.3 \\
\hline $3-1,132$ & 61.83 & 2.29 & -0.13 & 4.03 & -0.05 & 12.9 & 79.6 & 15.7 & 4.7 \\
\hline $3-2,30$ & 62.31 & 1.94 & 0.88 & 3.41 & 0.32 & 37.6 & 63.5 & 18.9 & 17.6 \\
\hline $3-2,52$ & 62.53 & 2.27 & 0.55 & 3.31 & 0.34 & 30.1 & 80.6 & 13.3 & 6.1 \\
\hline $3-2,80$ & 62.81 & 1.81 & 0.84 & 2.79 & 0.38 & 51.2 & 63.1 & 20.2 & 16.7 \\
\hline $3-2,105$ & 63.06 & 1.76 & 0.57 & 2.72 & 0.16 & 50.5 & 68.9 & 20.2 & 10.9 \\
\hline $3-2,132$ & 63.33 & 1.90 & 0.55 & 3.67 & 0.25 & 29.5 & 74.4 & 15.2 & 10.4 \\
\hline $3-3,30$ & 63.81 & 2.44 & 0.66 & 3.60 & -0.07 & 38.1 & 73.1 & 12.9 & 14.0 \\
\hline $3-3,52$ & 64.03 & 2.31 & 0.37 & - & - & 37.7 & 67.5 & 14.7 & 17.8 \\
\hline $3-3,80$ & 64.31 & 2.83 & 0.64 & 4.02 & 0.02 & 22.0 & 72.8 & 11.0 & 16.2 \\
\hline $3-3,105$ & 64.56 & 3.44 & -0.31 & 3.57 & -0.01 & 37.2 & 65.2 & 10.7 & 24.1 \\
\hline $3-3,132$ & 64.83 & 2.42 & 0.65 & 3.40 & 0.17 & 46.8 & 68.7 & 11.4 & 19.9 \\
\hline $3-4,30$ & 65.31 & 2.69 & 0.25 & 3.29 & 0.37 & 42.3 & 73.0 & 16.0 & 11.0 \\
\hline $3-4,52$ & 65.53 & 2.74 & -0.18 & 3.47 & 0.10 & 56.2 & 76.1 & 16.1 & 7.8 \\
\hline $3-4,80$ & 65.81 & 2.40 & -0.18 & 4.08 & -0.06 & 38.8 & 74.9 & 16.6 & 8.5 \\
\hline $3-4,105$ & 66.06 & 2.72 & -0.01 & 4.46 & -0.02 & 14.6 & 81.7 & 15.8 & 2.5 \\
\hline $3-4,132$ & 66.33 & 2.31 & 0.00 & - & - & 10.7 & 79.6 & 17.1 & 3.3 \\
\hline $3-5,30$ & 66.81 & 3.02 & 0.21 & - & - & 19.3 & - & - & - \\
\hline $3-5,52$ & 67.03 & 3.47 & 1.18 & - & - & 10.0 & 79.0 & 18.2 & 2.8 \\
\hline $3-5,80$ & 67.31 & 2.61 & 0.15 & - & - & 7.1 & 82.8 & 15.5 & 1.7 \\
\hline $3-5,105$ & 67.56 & 2.99 & 0.40 & 4.59 & -0.23 & 5.5 & 76.1 & 20.8 & 3.1 \\
\hline $3-5,132$ & 67.83 & 2.84 & 0.52 & 4.37 & -0.17 & 21.1 & 80.7 & 14.7 & 4.6 \\
\hline $3-6,30$ & 68.31 & 3.55 & 0.29 & 4.32 & -0.01 & 6.0 & 77.4 & 20.8 & 1.8 \\
\hline $4-1,30$ & 70.41 & 2.73 & -0.09 & $4.35^{*}$ & $-1.45^{*}$ & 15.8 & 79.4 & 15.1 & 5.5 \\
\hline $4-1,52$ & 70.63 & 3.14 & -0.19 & $4.13^{*}$ & $-0.39^{*}$ & 17.7 & 79.7 & 15.4 & 4.9 \\
\hline $4-1,80$ & 70.91 & 2.74 & -0.45 & - & - & 12.5 & 82.8 & 14.9 & 2.3 \\
\hline $4-1,105$ & 71.16 & 2.48 & 0.20 & - & - & 4.9 & 82.8 & 15.8 & 1.4 \\
\hline $4-1,132$ & 71.43 & 3.01 & 0.00 & 4.33 & -0.34 & 10.3 & 82.7 & 13.9 & 3.4 \\
\hline $4-2,30$ & 71.91 & 2.95 & -0.03 & 4.66 & 0.03 & 14.7 & 77.8 & 16.5 & 5.7 \\
\hline $4-2,52$ & 72.13 & 2.31 & 0.04 & - & - & 17.0 & 78.3 & 16.6 & 5.1 \\
\hline $4-2,80$ & 72.41 & 2.79 & 0.15 & - & - & 9.7 & 82.0 & 14.9 & 3.1 \\
\hline $4-2,105$ & 72.66 & 2.61 & -0.62 & 3.92 & -0.21 & 12.2 & 80.4 & 16.1 & 3.5 \\
\hline $4-2,132$ & 72.93 & 3.24 & 0.33 & - & - & 6.9 & - & - & - \\
\hline $5-3,30$ & 83.01 & 1.79 & 0.20 & 3.19 & 0.09 & 65.5 & 50.7 & 32.8 & 16.5 \\
\hline $5-3,52$ & 83.23 & 1.78 & -0.15 & 3.33 & 0.33 & 72.0 & 45.2 & 41.1 & 13.7 \\
\hline $5-3,80$ & 83.51 & 1.57 & 0.34 & 2.65 & 0.92 & 68.7 & 49.6 & 34.2 & 16.2 \\
\hline $5-3,105$ & 83.76 & 1.28 & 0.16 & 3.44 & 0.36 & 75.6 & 52.3 & 39.1 & 8.6 \\
\hline $5-3,132$ & 84.03 & 2.16 & 0.37 & 3.83 & 0.18 & 52.7 & 63.0 & 29.4 & 7.6 \\
\hline $5-4,30$ & 84.51 & 2.94 & 0.48 & - & - & 11.8 & 78.8 & 18.2 & 3.0 \\
\hline $5-4,52$ & 84.73 & 2.93 & 0.19 & - & - & 11.4 & 77.6 & 19.7 & 2.7 \\
\hline $5-4,80$ & 85.01 & - & - & 5.21 & -0.12 & 11.5 & 78.9 & 18.4 & 2.7 \\
\hline $5-4,105$ & 85.26 & 3.16 & 0.47 & - & - & 7.1 & 82.4 & 15.4 & 2.2 \\
\hline $5-4,132$ & 85.53 & 3.27 & 0.46 & - & - & 5.6 & 74.9 & 20.0 & 5.1 \\
\hline $5-5,30$ & 86.01 & 2.75 & 0.43 & - & - & 7.1 & 81.3 & 16.9 & 1.8 \\
\hline $5-5,52$ & 86.23 & 2.78 & 0.03 & 4.25 & -0.25 & 7.1 & 80.4 & 17.3 & 2.3 \\
\hline $5-5,80$ & 86.51 & 2.31 & 0.96 & 4.00 & -0.35 & 10.5 & 84.4 & 14.0 & 1.6 \\
\hline
\end{tabular}


Table 2. (Continued).

\begin{tabular}{|c|c|c|c|c|c|c|c|c|c|}
\hline \multirow{2}{*}{$\begin{array}{l}\text { Core-Section } \\
\text { (level in cm) }\end{array}$} & \multirow{2}{*}{$\begin{array}{l}\text { Corrected } \\
\text { sub-bottom } \\
\text { depth }(\mathrm{m})\end{array}$} & \multicolumn{2}{|c|}{$\begin{array}{l}\text { Planktonic } \\
\text { foraminifers }\end{array}$} & \multicolumn{2}{|c|}{$\begin{array}{l}\text { Benthic } \\
\text { foraminifers }\end{array}$} & \multirow{2}{*}{$\begin{array}{c}\mathrm{CaCO}_{3} \\
(\%)\end{array}$} & \multicolumn{3}{|c|}{ Texture $(\%)$} \\
\hline & & $\delta^{18} \mathrm{O}$ & ${ }^{\delta^{13} \mathrm{C}}$ & $\delta^{18} \mathrm{O}$ & ${ }_{\delta^{13} \mathrm{C}}$ & & Silt & Clay & Sand \\
\hline \multicolumn{10}{|c|}{ Hole 594A (Cont). } \\
\hline $5-5,105$ & 86.76 & 2.53 & 0.35 & 4.16 & -0.29 & 10.9 & 83.0 & 15.3 & 1.7 \\
\hline $5-5,132$ & 87.03 & 1.94 & 0.16 & 3.56 & -0.30 & 20.2 & 73.5 & 22.6 & 3.9 \\
\hline $5-6,30$ & 87.51 & 2.13 & 0.40 & 3.34 & 0.23 & 43.5 & 62.9 & 25.9 & 11.2 \\
\hline $5-6,52$ & 87.73 & 1.70 & 0.05 & 3.44 & 0.19 & 45.0 & 63.2 & 27.5 & 9.3 \\
\hline $5-6,80$ & 88.01 & 1.43 & 0.22 & 3.53 & -0.10 & 33.4 & 67.5 & 21.8 & 10.7 \\
\hline $5-6,105$ & 88.26 & 1.72 & 0.37 & 3.64 & 0.01 & 48.5 & 61.4 & 28.0 & 10.6 \\
\hline $5-6,132$ & 88.53 & 1.57 & 0.50 & 3.58 & -0.02 & 53.7 & 59.9 & 28.7 & 11.4 \\
\hline $6-1,30$ & 89.61 & 1.97 & 1.17 & 3.20 & -0.13 & 63.4 & 54.5 & 28.9 & 16.6 \\
\hline $6-1,52$ & 89.83 & 1.97 & 1.21 & 3.30 & -0.09 & 69.2 & 42.7 & 44.3 & 13.0 \\
\hline $6-1,80$ & 90.11 & 2.19 & 1.07 & 3.29 & 0.06 & 6.5 & 56.4 & 32.1 & 11.5 \\
\hline $6-1,105$ & 90.36 & 1.94 & 0.64 & 3.68 & 0.03 & - & 55.9 & 22.8 & 21.3 \\
\hline $6-1,132$ & 90.63 & 2.40 & 0.08 & 3.95 & -0.50 & - & 82.3 & 14.8 & 2.9 \\
\hline $6-2,30$ & 91.11 & 3.15 & 0.30 & 4.48 & -0.36 & - & 77.8 & 19.7 & 2.5 \\
\hline $6-2,52$ & 91.33 & 2.82 & 0.37 & 4.34 & -0.11 & 9.3 & 79.8 & 14.5 & 5.7 \\
\hline $6-2,80$ & 91.61 & 2.55 & 0.23 & 4.64 & -0.43 & 6.9 & 79.1 & 11.8 & 9.1 \\
\hline $6-2,105$ & 91.86 & 2.85 & 0.71 & 4.02 & -0.06 & 14.9 & 76.8 & 12.2 & 11.0 \\
\hline $6-2,132$ & 92.13 & 2.54 & 0.25 & 4.09 & -0.09 & 16.4 & 78.7 & 12.6 & 8.7 \\
\hline
\end{tabular}

Note: Planktonic foraminiferal measurements are on Globigerina bulloides (125-250 $\mu \mathrm{m})$; benthic measurements are on Uvigerina sp. (125-250 $\mu \mathrm{m})$, and, for asterisked values only, on Cibicides sp. (125-250 $\mu \mathrm{m})$. Where duplicate isotope results are shown for samples the average value has been plotted on Figs. 3 and 4 . Where benthic foraminiferal isotope values are not given, the samples contained too few suitable specimens for analysis. Details of isotope analytical procedures are recorded in Nelson, Hendy, and Dudley, (this volume). -, not determined.

til $25 \mathrm{~m}$ sub-bottom. According to Thierstein et al. (1977), E. huxleyi evolved about 0.27 m.y. ago, during isotope stage 8 . At Site 594 such an evolutionary age appears to be inconsistent with the interpretation of the isotope curve presented here, which in its central portion has been positioned on the basis of the $P$. lacunosa/S. universus bioevents defining isotope stage 12 (cf. Figs. 3 and 4). The distribution and age significance of $E$. huxleyi in the core require more detailed study, particularly as the subantarctic location of the site is known to have complicated the chronostratigraphic interpretation of several other microfossil zonal species in the core (Site 594 report, this volume).

The paleomagnetic and biostratigraphic data give average rates of sedimentation for the Brunhes normal Chron of $13.4 \mathrm{~cm} / 10^{3} \mathrm{yr}$. so an extremely high resolution stratigraphy is available compared with those of most deep-sea cores spanning the same time interval. Analytical results for 315 samples are included on Figure 3, with a sample interval of one sample every $30 \mathrm{~cm}$ of core, or a mean age resolution of one sample per $2400 \mathrm{yr}$.

\section{DISCUSSION OF RESULTS}

The summary stratigraphic log (Fig. 3) shows a remarkably consistent downcore correlation between the changes in sediment color represented by the alternating zones of pelagic and hemipelagic oozes, and each of the textural, carbonate, and isotope parameters analyzed. Despite rather wide variations in the absolute values of these properties, the pelagic sediments are always relatively enriched in sand- (dominantly foraminifers) and clay- (dominantly calcareous nannofossils and clay minerals) sized material, in calcium carbonate, in ${ }^{16} \mathrm{O}$ (lower $\delta^{18} \mathrm{O}$ values), and in ${ }^{13} \mathrm{C}$ (higher $\delta^{13} \mathrm{C}$ values) compared to the adjacent hemipelagic intervals. Typical ranges in values are given in Table 4 . The oxygen isotope data clearly indicate that the major pelagic intervals are associated with interglacial periods. By implication, therefore, the sediment properties of high carbonate content and increased sand fraction also equate with interglacial episodes. This association is opposite to that generally reported from Pacific cores (e.g., Hays, et al., 1969; Thompson and Saito, 1974; Shackleton and Opdyke, 1976; Valencia, 1977; Volat et al., 1980).

Griggs et al. (1983) have discussed the stratigraphy of short piston cores spanning the last $30 \times 10^{3} \mathrm{yr}$. or so from the same general area as Site 594. They developed a "sedimentary model" to explain the occurrence of contrasting sediment facies in their cores. We believe that this model can be extended back over at least the past $0.75 \mathrm{~m}$.y. to account for the interrelationships between texture, carbonate content, and oxygen isotope values documented here. During glacial periods of lowered sea level $\left({ }^{18} \mathrm{O}\right.$ enrichment), glaciation in the Southern Alps of South Island greatly increased the volume of terrigenous sediments delivered by rivers and wind to the east coast shelf and beyond, particularly the content of siltsized mica and quartz derived from a schist-dominated provenance. Micaceous hemipelagic sediments accumulated offshore with a proportionately reduced biogenic component dominated by diatoms, sponge spicules, and/ or radiolarians rather than planktonic foraminifers and coccolithophorids (Fig. 2). The preponderance of siliceous microfossils may be attributed to a greatly enhanced concentration of silicic acid (compared to nitrate) in Subantarctic Surface Waters (e.g., Zentara and Kamykowski, 1981) as a result of accelerated erosion of the adjacent landmass (Suggate et al., 1978), as well as generally lowered water temperatures and possibly increased dissolution of the carbonate microfossils (Griggs et al., 1983). 

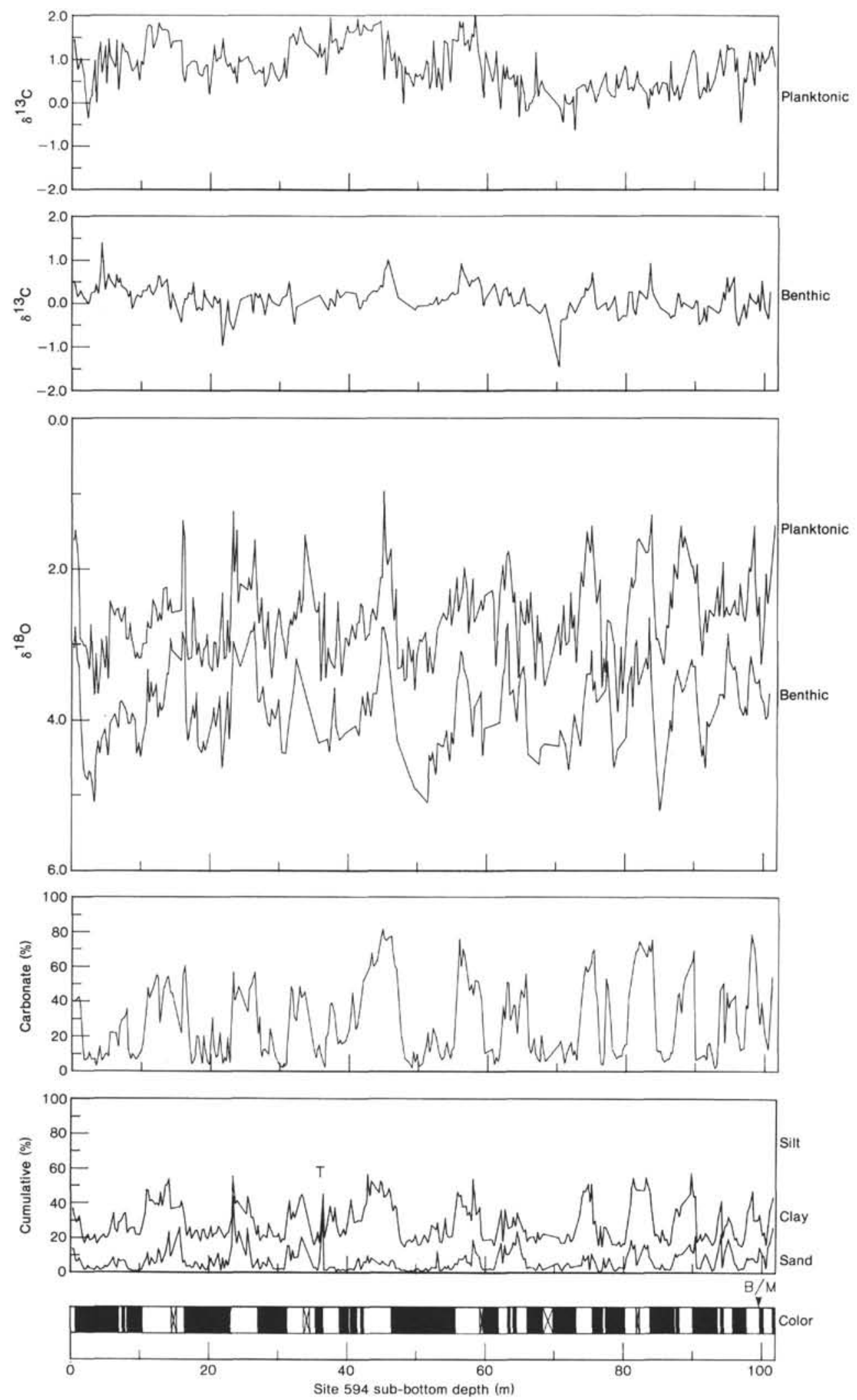

Figure 3. Summary stratigraphic logs of the color, texture, and calcium carbonate content of bulk core samples and of the oxygen and carbon isotope variations of planktonic (Globigerina bulloides) and benthic (Uvigerina sp.) foraminifers in the late Quaternary oozes at Site 594 (data from Table 2). B/M, Brunhes/Matuyama boundary $\left(730 \times 10^{3} \mathrm{yr}\right.$.) at $99.3 \mathrm{~m}$ sub-bottom depth. T, sand-rich tephra bed. The color variation is simplified: white intervals are dominantly bluish gray pelagic oozes; black intervals are mainly greenish gray hemipelagic oozes. Crosses indicate gaps in the cored sequence. Isotope values are in per mil deviations $(\%)$ from the international PDB standard. In this plot, the Cibicides isotope results (Table 2) have been converted to nominal Uvigerina values (Nelson, Hendy, and Dudley, this volume). 
Table 3. Distribution of some Quaternary zonal microfossil species at Site 594 and their possible age significance as adopted elsewhere.

\begin{tabular}{|c|c|c|c|c|c|c|}
\hline Index species & $\begin{array}{c}\text { Appearance } \\
\text { datum }\end{array}$ & $\begin{array}{l}\text { Suggested } \\
\text { age (m.y.) }\end{array}$ & $\begin{array}{l}\text { Isotope } \\
\text { stage }\end{array}$ & $\begin{array}{c}\text { Core } \\
\text { sample }\end{array}$ & $\begin{array}{l}\text { Sub-bottom } \\
\text { depth (m) }\end{array}$ & Abundance \\
\hline Emiliania huxleyi & First & 0.27 & 8 & $\begin{array}{l}6-1,25 \mathrm{~cm} \\
6-3,3 \mathrm{~cm}\end{array}$ & $\begin{array}{l}44.56 \\
47.34\end{array}$ & $\begin{array}{c}\text { Rare } \\
\text { Absent }\end{array}$ \\
\hline Stylatractus universus & Last & 0.41 & $11 / 12$ & $\begin{array}{l}7-1 \\
7.2\end{array}$ & $\begin{array}{l}-54.6 \\
-55.9\end{array}$ & $\begin{array}{c}\text { Absent } \\
\text { Rare }\end{array}$ \\
\hline Pseudoemiliania lacunosa & Last & 0.46 & 12 & $\begin{array}{l}7-1,3 \mathrm{~cm} \\
7-1, \mathrm{CC} \\
6-1,33 \mathrm{~cm}^{\mathrm{a}} \\
7-1,31 \mathrm{~cm}^{\mathrm{a}}\end{array}$ & $\begin{array}{l}53.94 \\
63.50 \\
44.63 \\
54.21\end{array}$ & $\begin{array}{l}\text { Absent } \\
\text { Rare } \\
\text { Absent } \\
\text { Present }\end{array}$ \\
\hline Calcidiscus macintyrei & Last & 1.45 & - & $\begin{array}{l}11-3,3 \mathrm{~cm} \\
11-5,3 \mathrm{~cm}\end{array}$ & $\begin{array}{l}95.34 \\
98.34\end{array}$ & $\begin{array}{c}\text { Absent } \\
\text { Rare }\end{array}$ \\
\hline
\end{tabular}

Note: Distributions from Site 594 site report, this volume; Lohman, this volume, and A. R. Edwards, pers. comm., 1983. Age significance after Hays and Shackleton, 1976; Gartner, 1977; Shackleton, 1977b; and Thierstein et al., 1977.

a From A. R. Edwards (pers. comm., 1983).

Table 4. Typical range of values of textural, carbonate, and isotope parameters for the late Quaternary pelagic and hemipelagic oozes at Site 594.

\begin{tabular}{lcc}
\hline \multicolumn{1}{c}{ Property } & Pelagic lithofacies & Hemipelagic lithofacies \\
\hline Sand sizes (\%) & $10-30$ & $0-10$ \\
Silt sizes $(\%)$ & $50-70$ & $70-90$ \\
Clay sizes $(\%)$ & $25-50$ & $10-25$ \\
$\mathrm{CaCO}_{3}(\%)$ & $40-80$ & $0-25$ \\
${ }^{18} \mathrm{O}(\% 0)$ & & \\
$\quad$ Planktonic foraminifers & $1.5-2.5$ & $2.5-3.5$ \\
$\quad$ Benthic foraminifers & $3.0-4.0$ & $4.0-5.0$ \\
${ }^{13} \mathrm{C}(\% 0)$ & $1.0-2.0$ & $0-1.0$ \\
$\quad$ Planktonic foraminifers & $0-1.0$ & $-0.5-0.5$ \\
$\quad$ Benthic foraminifers & & \\
\hline
\end{tabular}

During interglacial episodes and higher sea levels, erosion along the alpine chain was much less severe and the terrigenous sediment was mainly trapped on extensive piedmont plains or in glacial lakes. Apart from fine clays, the supply of terrigenous material to offshore basins was reduced. Coincidentally, there was a decrease in siliceous biogenic productivity and, according to Griggs et al. (1983), carbonate dissolution, with deposition of predominantly foraminifer-bearing nannofossil pelagic oozes (Fig. 2). Thus, the entire late Quaternary sedimentary record at Site 594 is dominated by paleoclimate influences that have controlled sea level and isotope composition, terrigenous input, calcium carbonate content, and biogenic productivity and composition. The three factors of dilution by noncarbonate material, calcareous plankton productivity, and intensity of carbonate dissolution together have determined the calcium carbonate stratigraphy at the site, but dilution by terrigenous and, to a lesser extent, siliceous microfossil materials is regarded as the dominant factor. Evidence for this can be gauged from Table 5, which shows that glacial isotope stages have overall sedimentation rates that were two to three times greater than interglacial isotope stages, but had calcium carbonate accumulation rates only about half those of the interglacial stages. Moreover, it is particularly relevant that the pulses of terrigenous sediment derived ultimately from glacial and periglacial erosion in the South Island have accompanied every period of relatively increased $\delta^{18} \mathrm{O}$ values corresponding to low- ered sea levels. This demonstrates a direct link throughout the last 0.75 m.y. between the fluctuations of the major Northern Hemisphere ice sheets and the advance and retreat of small alpine glaciers on a remote, temperate-latitude island in the South Pacific.

Tentative correlation between the planktonic and benthic $\delta^{18} \mathrm{O}$ fluctuations and the standard isotope stages is presented in Figure 4. For comparison, our $\delta^{18} \mathrm{O}$ isotope records are plotted alongside the planktonic foraminiferal record from DSDP Site 502 in the western Caribbean (Prell, 1982) as well as "SPECMAP"- a synthesis of the $\delta^{18} \mathrm{O}$ records from five cores (including DSDP Site 502) plotted as a function of time (Imbrie et al., in press). The isotope stage boundaries for DSDP Site 502 cores are from Prell (1982). It should be noted however, that Prell's positions for several of the stage boundaries at Site 502 in Figure 4 are at considerable variance with those given by Imbrie et al. (in press, table 6, column 7), which may be in error.

The proximity of Site 594 to the Subtropical Convergence and the very high sedimentation rate (average $13.4 \mathrm{~cm} / 10^{3} \mathrm{yr}$.), which has enabled a 30 -cm sampling interval, thus eliminating intersample mixing and reducing smoothing of the isotope signature by bioturbation (Shackleton and Opdyke, 1976), has resulted in some important differences between the Site $594 \delta^{18} \mathrm{O}$ record and those of most other cores penetrating the Brunhes/Matuyama boundary:

1. Some additional full glacial/interglacial cycles are recognizable in the late Quaternary. This is particularly apparent during isotope stage 15 (tentative correlation only), where the $\delta^{18} \mathrm{O}$ record twice reaches full glacial values during an interglacial stage, and in glacial stages 14 and 18 (tentative correlations only), where prominent interglacial $\delta^{18} \mathrm{O}$ peaks are recorded.

2. The magnitude of the glacial to interglacial $\delta^{18} \mathrm{O}$ fluctuations remains more or less constant at 2.0 to $2.2 \% 0$ throughout the entire Brunhes Chron and contrasts with the "SPECMAP" synthesis, which shows a decreasing $\delta^{18} \mathrm{O}$ amplitude with age. This latter is probably mainly a consequence of the diminishing resolution with age of the data base used by Imbrie et al. (in press).

3. The $\delta^{18} \mathrm{O}$ isotope record includes the common occurrence of short-term fluctuations (within a few to sev- 
Table 5. Tentative scheme of isotope stage positions and sedimentation rates for the late Quaternary oozes at Site 594.

\begin{tabular}{|c|c|c|c|c|c|c|c|c|}
\hline \multirow{2}{*}{\multicolumn{3}{|c|}{ Isotope stage boundary }} & \multicolumn{6}{|c|}{ Isotope stage } \\
\hline & & & & & & & & $\mathrm{CaCO}_{3}$ sed. \\
\hline Position & $\begin{array}{l}\text { Depth } \\
(\mathrm{cm})\end{array}$ & $\begin{array}{c}\mathrm{Age}^{\mathrm{a}} \\
\left(10^{3} \mathrm{yr}\right)\end{array}$ & Number & $\begin{array}{l}\text { Thickness } \\
(\mathrm{cm})\end{array}$ & $\begin{array}{l}\text { Duration } \\
\left(10^{3} \text { yr. }\right)\end{array}$ & $\begin{array}{c}\text { rate } \\
\left(\mathrm{cm} / 10^{3} \mathrm{yr}\right)\end{array}$ & $\begin{array}{l}\mathrm{CaCO}_{3} \\
\text { (mean \%) }\end{array}$ & $\begin{array}{c}\text { rate } \\
\left(\mathrm{cm} / 10^{3} \mathrm{yr} .\right)\end{array}$ \\
\hline \multirow{2}{*}{$1 / 2$} & 14 & 12 & 1 & 144 & 12 & 12.0 & 34.5 & 4.1 \\
\hline & 144 & 12 & 2 & 404 & 12 & 33.7 & 8.4 & 2.8 \\
\hline \multirow[t]{2}{*}{$2 / 3$} & 548 & 24 & & & & & & \\
\hline & & & 3 & 315 & 35 & 9.0 & 22.3 & 2.0 \\
\hline $3 / 4$ & 863 & 59 & 4 & 227 & 12 & 18.9 & 10.3 & 1.9 \\
\hline \multirow{2}{*}{$4 / 5$} & 1090 & 71 & & & & & & \\
\hline & 1660 & 128 & 5 & 570 & 57 & 10.0 & 45.6 & 4.6 \\
\hline $5 / 6$ & & & 6 & 640 & 58 & 11.0 & 12.7 & 1.4 \\
\hline \multirow{2}{*}{$6 / 7$} & 2300 & 186 & & & & & & \\
\hline & 2696 & 245 & 7 & 396 & 59 & 6.7 & 45.5 & 3.0 \\
\hline $7 / 8$ & 2070 & & 8 & 491 & 58 & 8.5 & 10.6 & 0.9 \\
\hline \multirow[t]{2}{*}{$8 / 9$} & 3187 & 303 & & & & & & \\
\hline & 3615 & 339 & 9 & 428 & 36 & 11.9 & 33.6 & 4.0 \\
\hline $9 / 10$ & & & 10 & 525 & 23 & 22.8 & 24.6 & 5.6 \\
\hline \multirow[t]{2}{*}{$10 / 11$} & 4140 & 362 & & & & & & \\
\hline & 4662 & 423 & 11 & 522 & 61 & 8.6 & 64.6 & 5.6 \\
\hline $11 / 12$ & & & 12 & 856 & 55 & 15.6 & 11.8 & 1.8 \\
\hline $12 / 13$ & 5518 & 478 & & & & & & \\
\hline \multirow[t]{2}{*}{$13 / 14$} & 5916 & 524 & 13 & 398 & 46 & 8.7 & 55.5 & 4.8 \\
\hline & 7334 & 565 & 14 & 1418 & 41 & 34.6 & 21.4 & 7.4 \\
\hline $14 / 15$ & 10304 & 302 & 15 & 1071 & 55 & 19.5 & 42.4 & 8.3 \\
\hline $15 / 16$ & 8405 & 620 & & & & & & \\
\hline \multirow[t]{2}{*}{$16 / 17$} & 8649 & 659 & 16 & 244 & 39 & 6.3 & 8.6 & 0.5 \\
\hline & 9074 & 689 & 17 & 425 & 30 & 14.2 & 39.4 & 5.6 \\
\hline $17 / 18$ & 3014 & 803 & 18 & 679 & 37 & 18.4 & 24.0 & 4.4 \\
\hline $18 / 19$ & 9753 & 726 & & & & & & \\
\hline $19 / 20$ & 9940 & 736 & 19 & 187 & 10 & 18.7 & 52.8 & 9.9 \\
\hline
\end{tabular}

${ }^{a}$ Based on Imbrie et al. (in press).

eral thousand years) with an amplitude of about $0.7 \%$. Some of these fluctuations occur concurrently in the planktonic and benthic records, which would suggest that high-frequency fluctuations in ice volumes and eustatic sea levels may be an intrinsic feature of the late Quaternary. In addition, a number of the planktonic shortterm fluctuations are not evident in the benthic record and may result from changes in surface water temperatures, perhaps associated with shifts in the location of the Subtropical Convergence over Site 594 (Fig. 1).

The foraminiferal $\delta^{13} \mathrm{C}$ record shows some significant variations and, as plotted, is broadly in phase with the $\delta^{18} \mathrm{O}$ curve (Fig. 3). Intervals of reduced $\delta^{13} \mathrm{C}$ values correspond to glacial episodes, while relatively higher $\delta^{13} \mathrm{C}$ values accompany interglacials. Near-surface waters in this region probably have $\delta^{13} \mathrm{C}$ values in the range 1.0 $2.0 \%$ for dissolved bicarbonate (Kroopnick, 1971; Kennett et al., 1979). This overlaps with the majority of interglacial values for the Globigerina bulloides calcite at the site and may indicate that the species deposited its carbonate close to carbon isotope equilibrium with dissolved bicarbonate. The Uvigerina calcite has $\delta^{13} \mathrm{C}$ values that are generally about $1 \% 0$ lower than those of $G$. bulloides and display a more subdued response to glacial/interglacial changes than do G. bulloides. Similar depletions of ${ }^{13} \mathrm{C}$ in planktonic and benthic foraminifers during glacial stages have been observed in other cores and have been interpreted by Shackleton (1977a) to result from a decrease in the organic carbon stored on land. Changes in the productivity of surface waters can also result in changes in the $\delta^{13} \mathrm{C}$ values of both planktonic and benthic foraminifers (Fontugne and Duplessy, 1981), although the effect of this is difficult to assess at Site 594 since decreased carbonate productivity during glacial stages is matched by increased siliceous microorganism productivity. The higher frequency fluctuations in $\delta^{13} \mathrm{C}$ probably do relate to short-term changes in primary productivity, which presently shows considerable spatial and temporal variability in response to nutrient availability, depth of vertical mixing, circulation patterns, and water transparency (Bradford, 1983).

\section{CONCLUSIONS}

1. The Brunhes normal Chron (last $730 \times 10^{3}$ yr.) at Site 594 off southeast New Zealand consists of a $99.3-\mathrm{m}$ thick sequence of oozes that alternate between pelagic 

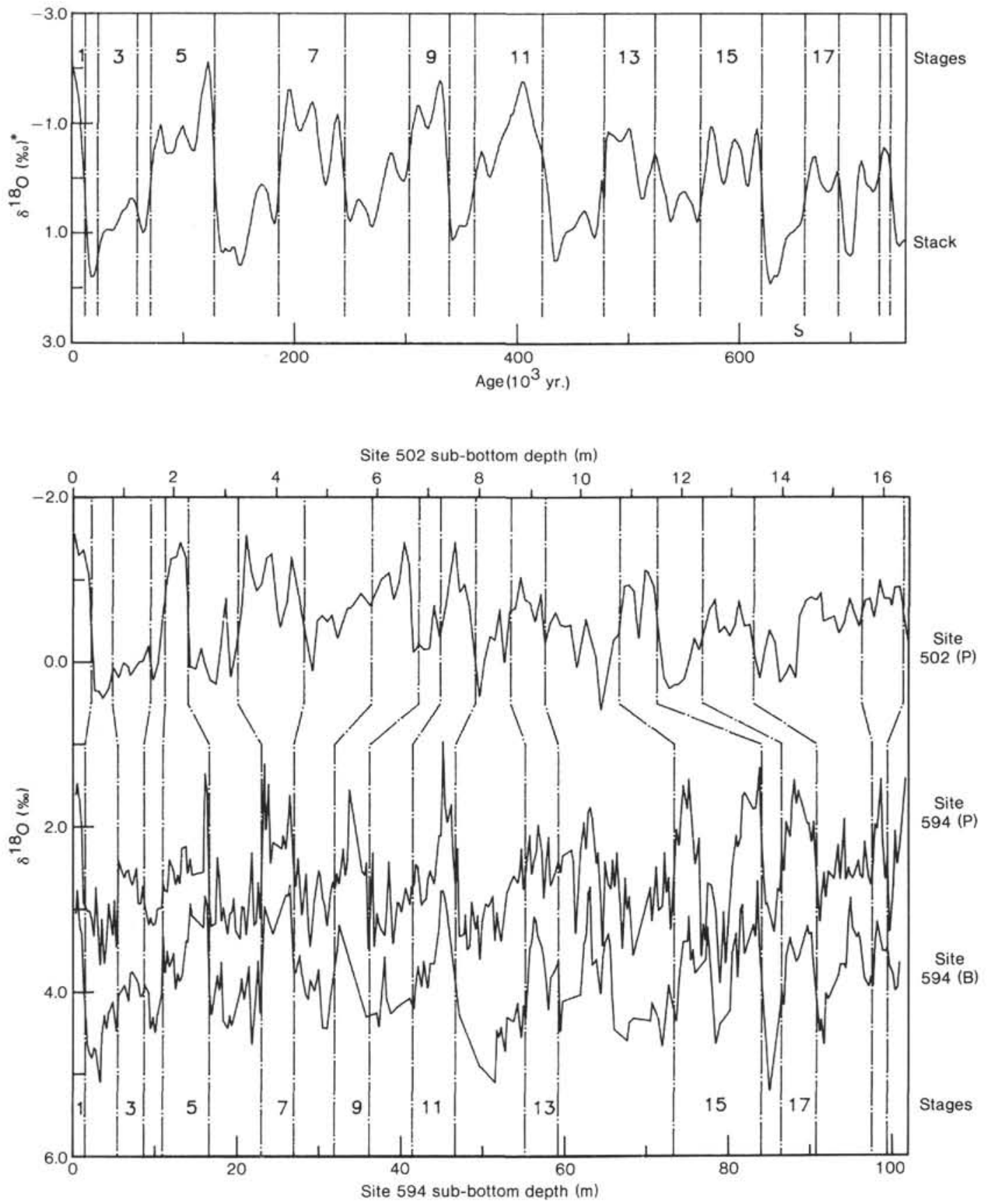

Figure 4. Tentative correlation scheme of late Quaternary isotope stages 1 to 19 defined for the age-based "SPECMAC" $\delta^{18} \mathrm{O}$ isotope stack (Imbrie et al., in press) and for the planktonic (P) foraminiferal $\delta^{18} \mathrm{O}$ record from DSDP Site 502 in the western Caribbean (Prell, 1982) with the planktonic (P) and benthic (B) foraminiferal $\delta^{18} \mathrm{O}$ records at DSDP Site 594, southeastern New Zealand. Note the significant difference in the sub-bottom depth scales for the same time period between DSDP Sites 502 and 594. "Note that the "SPECMAC" $\delta^{18} \mathrm{O}$ stack curve is expressed as standard deviation units (after Imbrie et al., in press).

and hemipelagic type as a result of paleoclimate fluctuations.

2. Hemipelagic sediments were deposited during glacial episodes and lowered eustatic sea level and are characterized by siliceous microfossils, low $(0-25 \%) \mathrm{CaCO}_{3}$ contents, and common terrigenous silt derived principally from accelerated physical erosion in South Island, New Zealand. Sedimentation rates at these times were typically $10-35 \mathrm{~cm} / 10^{3} \mathrm{yr}$.
3. Pelagic sediments formed during interglacial periods of high eustatic sea level and are characterized by calcareous microfossils, high $(50-80 \%) \mathrm{CaCO}_{3}$ contents, and a reduced terrigenous fraction that was predominantly clay-sized material. Sedimentation rates were typically $5-10 \mathrm{~cm} / 10^{3} \mathrm{yr}$.

4. The history of alpine glaciations and associated erosion in South Island, recorded by the hemipelagic ooze intervals, appears to be closely in phase with major fluc- 
tuations of the Pleistocene ice sheets as expressed by the influence of eustatic sea-level changes on the oxygen isotope composition of foraminifers in the core.

5. The Site 594 benthic and planktonic foraminiferal $\delta^{18} \mathrm{O}$ records include at least three additional full glacial-interglacial cycles compared to the standard isotope stage scheme for the late Quaternary. The intensity of glacial to interglacial fluctuations has remained similar throughout this entire period. Moreover, superimposed high-frequency $\delta^{18} \mathrm{O}$ fluctuations of $\sim 0.7 \%$ amplitude suggest that rapid eustatic sea-level oscillations of up to $50 \mathrm{~m}$ may be an important feature of the late Quaternary.

\section{ACKNOWLEDGMENTS}

We thank Scott Nodder and Steve Bergin (University of Waikato) for assistance with sample processing; Lionel Carter and Janet Bradford (N. Z. Oceanographic Institute, Wellington) for helpful discussions on the oceanography and bottom sediments off eastern South Island; Tony Edwards (N.Z. Geological Survey, Lower Hutt) for providing some preliminary information on calcareous nannoplankton in coretop samples; and Carolyn Beltz (University of Waikato) for SEM assistance. The review comments on this manuscript by Nick Pisias (Oregon State University, Corvallis) were much appreciated. Research was supported partly by Grant $83 / 125$ to C.S. Nelson from the N.Z. University Grants Committee.

\section{REFERENCES}

Bradford, J. M., 1983. Plankton and primary productivity in the vicinity of the Chatham Rise. N.Z. Oceanogr. Inst. Oceanogr. Summary, 21:1-14.

Fontugne, M. F., and Duplessy, J. C., 1981. Organic carbon isotopic fractionation by marine plankton in the temperature range -1 to $31^{\circ} \mathrm{C}$. Oceanologica Acta, 4:85-90.

Gartner, S., 1977. Calcareous nannofossil biostratigraphy and revised zonation of the Pleistocene. Mar. Micropaleontol., 2:1-25.

Griggs, G. B., Carter, L., Kennett, J. P., and Carter, R. V., 1983. Late Quaternary marine stratigraphy southeast of New Zealand. Geol. Soc. Am. Bull., 94:791-797.

Hays, J. D., Saito, T., Opdyke, N. D., and Burckle, L. H., 1969. Pliocene-Pleistocene sediments of the equatorial Pacific: their paleomagnetic, biostratigraphic and climatic record. Geol. Soc. Am. Bull., 80:1481-1514.

Hays, J. D., and Shackleton, N. J., 1976. The globally synchronous extinction of Stylatractus universus. Geology, 4:649-652.

Healy, T. R., Nelson, C. S., de Lange, W., and McArthur, W., 1983. The University of Waikato rapid sediment analysis system for particle size analysis. Geol. Soc. N.Z. Misc. Publ., 30A:57.
Imbrie, J., Shackleton, N. J., Pisias, N. G., Morley, J. J., Prell, W. L., Martinson, D. G., Hays, J. D., McIntyre, A., and Mix, A. C., in press. The orbital theory of Pleistocene climate: support from a revised chronology of the marine $\delta^{18} \mathrm{O}$ record. In Imbrie, J., and Berger, A. (Eds.), Milankovitch and Climate Change: Amsterdam (Elsevier).

Kennett, J. P., Shackleton, N. J., Margolis, S. V., Goodney, D. E., Dudley, W. C., and Kroopnick, P. M., 1979. Late Cenozoic oxygen and carbon isotopic history and volcanic ash stratigraphy: DSDP Site 284, South Pacific. Am. J. Sci., 179:52-69.

Kroopnick, P. M., 1971. Oxygen and carbon in the oceans and atmosphere: stable isotopes as tracers for consumption, production, and circulation models [Ph.D. dissert.]. University of California, San Diego.

Prell, W. L., 1982. Oxygen and carbon isotope stratigraphy for the Quaternary of Hole 502B: evidence for two modes of isotopic variability. In Prell, W. L., Gardner, J. V., et al., Init. Repts. DSDP, 68: Washington (U.S. Govt. Printing Office), 455-464.

Shackleton, N. J., 1977a. Carbon-13 in Uvigerina: tropical rainforest history and equatorial Pacific carbonate dissolution cycles. In Anderson, N. R., and Malahoff, A. (Eds.), The Fate of Fossil Fuel $\mathrm{CO}_{2}$ in the Oceans: New York (Plenum Press), pp. 401-427.

, 1977b. Oxygen isotope stratigraphy of the middle Pleistocene. In Shotton, F. W. (Ed.), British Quaternary Studies-Recent Advances: Oxford (Clarendon Press), pp. 1-16.

Shackleton, N. J., and Opdyke, N. D., 1976. Oxygen isotope and paleomagnetic stratigraphy of Pacific core V28-239, late Pliocene to latest Pleistocene. In Cline, R. M., and Hays, J. D. (Eds.), Investigation of Late Quaternary Paleoceanography and Paleoclimatology. Geol. Soc. Am. Mem., 145:449-464.

Suggate, R. P., Stevens, G. R., and Te Punga, M. T. (Eds.), 1978. The Geology of New Zealand, 2. New Zealand Geological Survey, Department of Scientific and Industrial Research, Wellington.

Theirstein, H. R., Geitzenauer, K. R., Molfino, B., and Shackleton, N. J., 1977. Global synchroneity of late Quaternary coccolith datum levels: validation by oxygen isotopes. Geology, 5:400-404.

Thompson, P. R., and Saito, T., 1974. Pacific Pleistocene sediments: planktonic foraminifera dissolution cycles and geochronology. Geology, 2:333-335.

Valencia, M. J., 1977. Pacific Pleistocene paleoclimatic stratigraphies: a comparative analysis of results. Quat. Res., 8:339-354.

Volat, J.-L., Pastouret, L., and Vergnaud-Grassini, C., 1980. Dissolution and carbonate fluctuations in Pleistocene deep-sea cores: a review. Mar. Geol., 34:1-28.

Zentara, S. J., and Kamykowski, D., 1981. Geographic variations in the relationship between silicic acid and nitrate in the South Pacific Ocean. Deep-Sea Res., 28A:455-465.

Date of Initial Receipt: 8 June 1984

Date of Acceptance: 5 December 1984 